\title{
De Sitter vacua of strongly interacting QFT
}

\author{
Alex Buchel ${ }^{a, b, c}$ and Aleksandr Karapetyan ${ }^{a}$ \\ ${ }^{a}$ Department of Applied Mathematics, University of Western Ontario, \\ London, Ontario N6A 5B\%, Canada \\ ${ }^{b}$ Department of Physics and Astronomy, University of Western Ontario, \\ London, Ontario N6A 5B\%, Canada \\ ${ }^{c}$ Perimeter Institute for Theoretical Physics, \\ Waterloo, Ontario N2J 2W9, Canada \\ E-mail: abuchel@uwo.ca, kaleksan@uwo.ca
}

ABStRaCt: We use holographic correspondence to argue that Euclidean (Bunch-Davies) vacuum is a late-time attractor of the dynamical evolution of quantum gauge theories at strong coupling. The Bunch-Davies vacuum is not an adiabatic state, if the gauge theory is non-conformal - the comoving entropy production rate is nonzero. Using the $\mathcal{N}=2^{*}$ gauge theory holography, we explore prospects of explaining current accelerated expansion of the Universe as due to the vacuum energy of a strongly coupled QFT.

KEYwords: AdS-CFT Correspondence, Gauge-gravity correspondence, Holography and quark-gluon plasmas

ARXIV EPRINT: 1702.01320 


\section{Contents}

1 Introduction $\quad 1$

$2 \mathcal{N}=2^{*}$ holography 3

$2.1 \mathcal{N}=2^{*}$ gauge theory 4

2.2 PW effective action 5

3 Holographic evolution of $\mathcal{N}=2^{*}$ gauge theory states in $d S_{4} \quad 6$

4 Vacuum of $\mathcal{N}=2^{*}$ gauge theory in $d S_{4} \quad 10$

$4.1 d S_{4}$ vacuum is Euclidean $\quad 12$

$4.2 \mathcal{E}_{v}$ of $\mathcal{N}=2^{*}$ gauge theory in $d S_{4} \quad 13$

$\begin{array}{lll}4.3 & d S_{4} \text { vacuum of a non-conformal gauge theory is non-adiabatic } & 15\end{array}$

5 Conclusion $\quad 18$

$\begin{array}{ll}\text { A Apparent horizon area growth theorem } & 21\end{array}$

B Mapping QFT dynamics in Minkowski and FLRW space-times 22

C $\mathcal{N}=4 \mathrm{SYM}$ in FLRW 24

\section{Introduction}

One of the outstanding questions of cosmology is the physics behind the current accelerated expansion of the Universe $[1,2]$. We argue that there is a strong coupling aspect of this question, which can be addressed using the holographic correspondence between strongly coupled gauge theories and dual gravitational models in asymptotically anti-de-Sitter (AdS) space-times $[3,4]$.

In a framework of classical Einstein gravity and quantum field theory, the dynamics of the Universe is described by the following equation

$$
G_{\mu \nu}=8 \pi G_{N}\left\langle T_{\mu \nu}\right\rangle
$$

where $G_{\mu \nu}$ is the Einstein tensor of the four-dimensional space-time metric $g_{\alpha \beta}^{(4)}, G_{N}=M_{p l}^{-2}$ is Newton's constant, and $\left\langle T_{\mu \nu}\right\rangle=\left\langle T_{\mu \nu}\left[g_{\alpha \beta}^{(4)}\right]\right\rangle$ is the quantum expectation value of the stressenergy tensor of all matter fields, self-consistently computed in the space-time background metric $g_{\alpha \beta}^{(4)}$. Gravitational Bianchi identity implies covariant conservation of the stressenergy tensor:

$$
\nabla^{\mu}\left\langle T_{\mu \nu}\right\rangle=0
$$


At late times, $t \rightarrow \infty$, expansion of the Universe will ultimately dilute all the components of the stress energy tensor, except for the vacuum energy,

$$
\lim _{t \rightarrow \infty}\left\langle T_{\mu \nu}\right\rangle=\left\langle\operatorname{vacuum}\left|T_{\mu \nu}\right| \operatorname{vacuum}\right\rangle=\operatorname{diag}\left(\mathcal{E}_{v}, P_{v}, P_{v}, P_{v}\right),
$$

where $\mathcal{E}_{v}$ and $P_{v}$ are the matter fields vacuum energy density and pressure correspondingly, where, using (1.2),

$$
\mathcal{E}_{v}+P_{v}=0 .
$$

If $\mathcal{E}_{v}>0$, the (spatially-flat) Universe at late-times is de Sitter, with a Hubble constant $H$ :

$$
d s_{4}^{2}=g_{\alpha \beta}^{(4)} d x^{\alpha} d x^{\beta}=-d t^{2}+a(t)^{2} d \boldsymbol{x}^{2}, \quad a(t)=e^{H t},
$$

such that

$$
H^{2} M_{p l}^{2}=\frac{8 \pi}{3} \mathcal{E}_{v}(H)
$$

where we explicitly indicated that the vacuum energy of the Standard Model (SM) of particle physics should be computed self-consistently in (1.5). Values of $H$ obtained from solving (1.6) can be reliable in so far as approximation (1.1) is valid, i.e., $H \ll M_{p l}$, so that the effects of quantum gravity can be neglected.

Computation of a vacuum energy of a QFT in curved space-time is a subject with a long history [5]. Almost all analysis are done though in the framework of perturbative weakly-coupled QFT. Irrespectively of the physics beyond the SM, the SM itself contains a sector with a strongly coupled sector in the infrared - namely, quantum chromodynamics (QCD). This is the main motivating factor of the present project - we use the holographic correspondence to compute the vacuum energy of certain strongly coupled gauge theories in de Sitter space-time.

Holographic computations of de Sitter vacuum energy of conformal field theories at strong coupling was done previously in [6]. However, because the theory in question was conformal, $\mathcal{E}_{v} \sim H^{4}$, since the Hubble constant is the only scale parameter in the model. Necessarily, (1.6) implies $H \sim M_{p l}$ which invalidates classical treatment of gravity in this regime. To have a chance of a self-consistent treatment of a classical Einstein gravity coupled to quantum SM, the latter should be modeled as a non-conformal gauge theory in a holographic setting. For one reason, QCD is responsible for a strongly coupled vacuum in our Universe precisely because it is non-conformal. Second, existence of an intrinsic scale $\Lambda$ in quantum gauge theory allows for a potential hierarchy between the scales $H$ and $M_{p l}$ generated in a relation (1.6).

Non-conformal gauge theories in de Sitter background were studied in holographic framework earlier [7-14]. The basic idea was to take a holographic correspondence describing a renormalization group (RG) flow for a vacuum state of a strongly coupled nonconformal gauge theory in Minkowski space-time and "deform" it to a background de Sitter geometry, $\mathbb{R}^{3,1} \rightarrow d S_{4}$. The resulting de Sitter RG flow, identified as a vacuum state of the corresponding gauge theory in $d S_{4}$, exhibited the $\mathrm{SO}(4,1)$ symmetry and allowed for a Euclidean analytical continuation. Thus, all the vacua discussed in [7-12] are Euclidean or Bunch-Davies [15]. It was recognized in $[16,17]$ (MA) that the vacuum state invariance 
under the proper de Sitter transformations in free field theories allows for a two-parameter family of inequivalent vacua. These MA-vacua are interesting in that they might lead to enhanced, order $\propto \frac{H}{M_{p l}}$, effects during inflation [18]. Subsequent studies of perturbative interactions in MA-vacua revealed various problems [19-22]. To our knowledge, no corresponding studies at strong coupling were ever performed. As a second motivation for our project, we use holographic framework to study the evolution of a generic spatially homogeneous and isotropic state in strongly coupled non-conformal gauge theory. We find that while at any finite boundary time $t$ the state is not invariant under $d S_{4}$ isometries, in the infinite future $t \rightarrow \infty$ the state evolves to a Bunch-Davies vacuum, equivalent to the ones discussed in [7-12]. A corollary of our analysis is that MA-vacua can not be reached dynamically from homogeneous and isotropic initial conditions in strongly coupled gauge theories with a holographic dual. This further suggests that MA-vacua are inconsistent in interacting quantum gauge theories.

There are several ways to generate non-conformal RG flows in holography. One can start with $\mathcal{N}=4$ superconformal Yang-Mills theory $A d S_{5} \times S^{5}$ duality [3] and deform it by turning on a nonzero coupling for a relevant operator, explicitly breaking the scale invariance of the parent theory. A typical representative here is the $\mathcal{N}=2^{*}$ holography [23-25]. Alternatively, the scale invariance of the parent theory can be broken spontaneously, as it is in the case of Klebanov-Strassler (KS) cascading gauge theory [26]. Finally, holography for a non-conformal gauge theory in four space-times dimensions can be obtained from the Kaluza-Klein reduction of the higher-dimensional scale invariant gauge theory/gravity correspondence, as in [27]. In this paper we focus on the $\mathcal{N}=2^{*}$ holographic correspondence and delegate the discussion of the KS theory to future work. ${ }^{1}$

The rest of the paper is organized as follows. In the next section we briefly review the holographic duality between $\mathcal{N}=2^{*}$ supersymmetric $\mathrm{SU}(N)$ gauge theory [24] and its Pilch-Warner (PW) gravitational dual [23]. In section 3, building on [29], we discuss evolution of generic homogeneous and isotropic states of $\mathcal{N}=2^{*}$ gauge theory in $d S_{4}$. In section 4 we show that at late times these states approach a Bunch-Davies (Euclidean) vacuum of the theory constructed in [9]. We argue that the Euclidean vacuum of nonconformal gauge theories is not an adiabatic state - the comoving entropy continues to be produced. We compute the vacuum energy $\mathcal{E}_{v}=\mathcal{E}_{v}(H)$ and the entropy production rate of strongly coupled $\mathcal{N}=2^{*}$ gauge theory in $d S_{4}$. We conclude in section 5 with speculations regarding explanation of the current accelerated expansion of the Universe due to a vacuum energy of a gauge theory sector at strong coupling. ${ }^{2}$

\section{$2 \mathcal{N}=2^{*}$ holography}

$\mathcal{N}=2^{*}$ holography is a correspondence between mass-deformed $\mathcal{N}=4 \mathrm{SU}(N)$ supersymmetric Yang-Mill theory at strong coupling and a PW five-dimensional $\mathcal{N}=2$ supergravity - a particular consistent truncation of ten-dimensional type IIb supergravity.

\footnotetext{
${ }^{1}$ Unlike non-conformal gauge theories obtained from compactifications of the higher-dimensional conformal gauge theories, KS cascading gauge theory is intrinsically four-dimensional [28].

${ }^{2}$ This proposal originated from discussions with David Mateos and Jorge Casalderrey-Solana.
} 


\section{$2.1 \mathcal{N}=2^{*}$ gauge theory}

In the language of four-dimensional $\mathcal{N}=1$ supersymmetry, the mass deformed $\mathcal{N}=4$ $\mathrm{SU}(N)$ Yang-Mills theory $\left(\mathcal{N}=2^{*}\right)$ in $\mathbb{R}^{3,1}$ consists of a vector multiplet $V$, an adjoint chiral superfield $\Phi$ related by $\mathcal{N}=2$ supersymmetry to the gauge field, and two additional adjoint chiral multiplets $Q$ and $\tilde{Q}$ which form an $\mathcal{N}=2$ hypermultiplet. In addition to the usual gauge-invariant kinetic terms for these fields, ${ }^{3}$ the theory has additional interactions and a hypermultiplet mass term given by the superpotential

$$
W=\frac{2 \sqrt{2}}{g_{Y M}^{2}} \operatorname{Tr}([Q, \tilde{Q}] \Phi)+\frac{m}{g_{Y M}^{2}}\left(\operatorname{Tr} Q^{2}+\operatorname{Tr} \tilde{Q}^{2}\right) .
$$

When $m=0$, the gauge theory is superconformal with $g_{Y M}$ characterizing an exactly marginal deformation. The theory has a classical $3(N-1)$ complex dimensional moduli space, which is protected by supersymmetry against (non)-perturbative quantum corrections.

When $m \neq 0$, the $\mathcal{N}=4$ supersymmetry is softly broken to $\mathcal{N}=2$. This mass deformation lifts the $\{Q, \tilde{Q}\}$ hypermultiplet moduli directions, leaving the $(N-1)$ complex dimensional Coulomb branch of the $\mathcal{N}=2 \mathrm{SU}(N)$ Yang-Mills theory, parameterized by expectation values of the adjoint scalar

$$
\Phi=\operatorname{diag}\left(a_{1}, a_{2}, \cdots, a_{N}\right), \quad \sum_{i} a_{i}=0
$$

in the Cartan subalgebra of the gauge group. For generic values of the moduli $a_{i}$, the gauge symmetry is broken to that of the Cartan subalgebra $\mathrm{U}(1)^{N-1}$, up to the permutation of individual U(1) factors. Additionally, the superpotential (2.1) induces the RG flow of the gauge coupling. While from the gauge theory perspective it is straightforward to study this $\mathcal{N}=2^{*}$ theory at any point on the Coulomb branch [30], the PW supergravity flow [23] corresponds to a particular Coulomb branch vacuum. More specifically, matching the probe computation in gauge theory and the dual PW supergravity flow, it was argued in [24] that the appropriate Coulomb branch vacuum corresponds to a linear distribution of the vevs $(2.2)$ as

$$
a_{i} \in\left[-a_{0}, a_{0}\right], \quad a_{0}^{2}=\frac{m^{2} g_{Y M}^{2} N}{\pi},
$$

with (continuous in the large $N$ limit) linear number density

$$
\rho(a)=\frac{2}{m^{2} g_{Y M}^{2}} \sqrt{a_{0}^{2}-a^{2}}, \quad \int_{-a_{0}}^{a_{0}} d a \rho(a)=N .
$$

The special character of the Coulomb branch vacuum (2.4) was explained in [31], where it was shown that this vacuum is a saddle point of the supersymmetric localization of $\mathcal{N}=2^{*}$ gauge theory on $S^{4}$ ( in the $S^{4}$ decompactification limit ) in the planar limit, i.e., $N \rightarrow \infty$ and $g_{Y M}^{2} \rightarrow 0$ with the 't Hooft coupling $N g_{Y M}^{2}=$ const $\gg 1$.

\footnotetext{
${ }^{3}$ The classical Kähler potential is normalized according to $\left(2 / g_{Y M}^{2}\right) \operatorname{Tr}[\bar{\Phi} \Phi+\bar{Q} Q+\overline{\tilde{Q}} \tilde{Q}]$.
} 


\subsection{PW effective action}

The gravitational dual to $\mathcal{N}=2^{*}$ supersymmetric gauge theory in the planar limit and for large 't Hooft coupling $N g_{Y M}^{2} \gg 1$ was constructed in [23]. A consistent truncation from type IIb supergravity is encoded in five-dimensional effective action

$$
\begin{aligned}
S & =\int_{\mathcal{M}_{5}} d \xi^{5} \sqrt{-g} \mathcal{L}_{5} \\
& =\frac{1}{16 \pi G_{5}} \int_{\mathcal{M}_{5}} d \xi^{5} \sqrt{-g}\left[R-12(\partial \alpha)^{2}-4(\partial \chi)^{2}-\mathcal{P}\right],
\end{aligned}
$$

where the potential ${ }^{4}$

$$
\mathcal{P}=\frac{1}{4}\left[\frac{1}{3}\left(\frac{\partial W}{\partial \alpha}\right)^{2}+\left(\frac{\partial W}{\partial \chi}\right)^{2}\right]-\frac{4}{3} W^{2}
$$

is a functional of supergravity scalars $\alpha$ and $\chi$, and is determined by the superpotential

$$
W=-e^{-2 \alpha}-\frac{1}{2} e^{4 \alpha} \cosh (2 \chi) .
$$

In our conventions, the five-dimensional Newton's constant is

$$
G_{5} \equiv \frac{G_{10}}{2^{5} \operatorname{vol}_{S^{5}}}=\frac{4 \pi}{N^{2}} .
$$

Effective action (2.5) can be further consistently truncated to $\{\chi=0, \alpha \neq 0\}$ or to $\{\alpha=\chi=0\}$. In the later case the vacuum solution is that of $A d S_{5}$, holographically dual to a vacuum of $\mathcal{N}=4 \mathrm{SYM}$.

The gravitational scalars $\alpha$ and $\chi$ are dual to dimension-two $\mathcal{O}_{2}$ and dimension-three $\mathcal{O}_{3}$ operators of $\mathcal{N}=4 \mathrm{SYM}$,

$$
\begin{aligned}
\alpha \Longleftrightarrow \mathcal{O}_{2}= & \frac{1}{3} \operatorname{Tr}\left(\left|\phi_{1}\right|^{2}+\left|\phi_{2}\right|^{2}-2\left|\phi_{3}\right|^{2}\right), \\
\chi \Longleftrightarrow \mathcal{O}_{3}= & -\operatorname{Tr}\left(i \psi_{1} \psi_{2}-\sqrt{2} g_{Y M} \phi_{3}\left[\phi_{1}, \phi_{1}^{\dagger}\right]+\sqrt{2} g_{Y M} \phi_{3}\left[\phi_{2}^{\dagger}, \phi_{2}\right]+\text { h.c. }\right) \\
& +\frac{2}{3} m_{f} \operatorname{Tr}\left(\left|\phi_{1}\right|^{2}+\left|\phi_{2}\right|^{2}+\left|\phi_{3}\right|^{2}\right),
\end{aligned}
$$

where $\phi_{1,2}$ are the bosonic components of the $\mathcal{N}=2$ hypermultiplet chiral superfields $\{Q, \tilde{Q}\}$, and $\phi_{3}$ is a bosonic component of the chiral superfield $\Phi ; \psi_{i}$ are the fermionic superpartners of $\phi_{i}$. The fermionic mass term $m_{f}$ and the bosonic mass term $m_{b}^{2}$ (the nonnormalizable coefficients of $\chi$ and $\alpha$ near the $A d S_{5}$ boundary correspondingly) realize the $\mathcal{N}=4$ SYM theory deformation

$$
\delta \mathcal{L}=-2 \int d^{4} x\left[m_{b}^{2} \mathcal{O}_{2}+m_{f} \mathcal{O}_{3}\right] .
$$

\footnotetext{
${ }^{4}$ We set the five-dimensional gauged supergravity coupling to one. This corresponds to setting the radius $L$ of the five-dimensional sphere in the undeformed metric to 2 .
} 
For a generic choice $m_{f}^{2} \neq m_{b}^{2}$ this deformation completely breaks the supersymmetry; when $m_{f}^{2}=m_{b}^{2} \equiv m^{2}, \delta \mathcal{L}$ is the mass deformation in the $\mathcal{N}=2$ supersymmetric superpotential (2.1).

PW effective action (2.5) does not reproduce the free energy of $\mathcal{N}=2^{*}$ gauge theory on $S^{4}$ computed from the supersymmetric localization in the $S^{4}$ decompactification limit [32]. Rather, additional couplings to the background $S^{4}$ metric are needed [33], resulting in an enlarged gravitational dual [34] (BEFP). PW effective action is a consistent truncation of the BEFP effective action. As outlined in the introduction, we are interested in the dynamics of $\mathcal{N}=2^{*}$ gauge theory in $d S_{4}$. Since the gauge theory background space-time of interest is curved, the BEFP effective action encoding additional curvature couplings, allows for a more general analysis. For simplicity, in this paper we limit the scope of study to $\mathrm{PW}$ truncation. ${ }^{5}$

\section{Holographic evolution of $\mathcal{N}=2^{*}$ gauge theory states in $d S_{4}$}

We use the characteristic formulation of gravitational dynamics in asymptotically AdS space-times summarized in [36] to describe evolution of spatially homogeneous and isotropic states of $\mathcal{N}=2^{*}$ gauge theory in $d S_{4}$. We follow closely the discussion in [29].

A generic state of the gauge theory, homogeneous and isotropic in the spatial boundary coordinates $\boldsymbol{x}=\{x, y, z\}$, leads to a dual gravitational metric ansatz

$$
d s_{5}^{2}=2 d t(d r-A d t)+\Sigma^{2} d \boldsymbol{x}^{2},
$$

with the warp factors $A, \Sigma$ as well as the bulk scalars $\alpha, \chi$ depending only on $\{t, r\}$. From PW effective action (2.5) we obtain the following equations of motion:

$$
\begin{aligned}
& 0=d_{+}^{\prime} \Sigma+2 \Sigma^{\prime} d_{+} \ln \Sigma+\frac{\Sigma}{6} \mathcal{P}, \\
& 0=A^{\prime \prime}-6(\ln \Sigma)^{\prime} d_{+} \ln \Sigma+4 \chi^{\prime} d_{+} \chi+12 \alpha^{\prime} d_{+} \alpha-\frac{\mathcal{P}}{6}, \\
& d_{+}^{\prime} \alpha+\frac{3}{2}\left((\ln \Sigma)^{\prime} d_{+} \alpha+\alpha^{\prime} d_{+} \ln \Sigma\right)-\frac{1}{48} \partial_{\alpha} \mathcal{P}, \\
& 0=d_{+}^{\prime} \chi+\frac{3}{2}\left((\ln \Sigma)^{\prime} d_{+} \chi+\chi^{\prime} d_{+} \ln \Sigma\right)-\frac{1}{16} \partial_{\chi} \mathcal{P},
\end{aligned}
$$

as well as the Hamiltonian constraint equation:

$$
0=\Sigma^{\prime \prime}+\left(4\left(\alpha^{\prime}\right)^{2}+\frac{4}{3}\left(\chi^{\prime}\right)^{2}\right) \Sigma
$$

and the momentum constraint equation:

$$
\begin{aligned}
0= & d_{+}^{2} \Sigma-2 A \Sigma^{\prime}-\left(4 A \Sigma^{\prime}+A^{\prime} \Sigma\right) d_{+} \ln \Sigma \\
& +\left(4\left(d_{+} \alpha\right)^{2}+\frac{4}{3}\left(d_{+} \chi\right)^{2}\right) \Sigma-\frac{1}{3} \Sigma A \mathcal{P} .
\end{aligned}
$$

\footnotetext{
${ }^{5}$ It would be interesting to explore the parameter space of the curvature couplings, and study the stability of the PW truncation within BEFP for $\mathcal{N}=2^{*}$ gauge theory formulated in curved space-time, extending stability analysis for thermal states of $\mathcal{N}=2^{*}$ plasma in Minkowski space-time [35].
} 
In (3.2)-(3.4) we denoted ${ }^{\prime}=\frac{\partial}{\partial r}, \cdot=\frac{\partial}{\partial t}$, and $d_{+}=\frac{\partial}{\partial t}+A \frac{\partial}{\partial r}$. The near-boundary $r \rightarrow \infty$ asymptotic behaviour ${ }^{6}$ of the metric function and the scalars encode the mass parameters $m_{b}^{2}$ and $m_{f}$ of the $\mathcal{N}=2^{*}$ gauge theory [37], and the boundary metric (1.5) scale factor $a(t)$ :

$$
\begin{aligned}
\Sigma & =\frac{a r}{2}+\mathcal{O}\left(r^{-1}\right), & A & =\frac{r^{2}}{8}-\frac{\dot{a} r}{a}+\mathcal{O}\left(r^{0}\right), \\
\alpha & =-\frac{8 m_{b}^{2} \ln r}{3 r^{2}}+\mathcal{O}\left(r^{-2}\right), & \chi & =\frac{2 m_{f}}{r}+\mathcal{O}\left(r^{-2}\right) .
\end{aligned}
$$

An initial state of the gauge theory is specified providing the scalar profiles $\alpha(0, r)$ and $\chi(0, r)$ and solving the constraint (3.3), subject to the boundary conditions (3.5). Equations (3.2) can then be used to evolve the state. Note that the gravitational bulk metric (3.1) does not enjoy the $\mathrm{SO}(4,1)$ boundary metric isometry.

The subleading terms in the boundary expansion of the metric functions and the scalars encode the evolution of the energy density $\mathcal{E}(t)$, pressure $P(t)$ and the expectation values of the operators $\mathcal{O}_{2}(t)$ and $\mathcal{O}_{3}(t)$ of the prescribed gauge theory initial state. Specifically, extending to asymptotic expansion (3.5) for $\{\alpha, \chi, A\}$,

$$
\begin{gathered}
\alpha=-\frac{8 m_{b}^{2} \ln r}{3 r^{2}}+\frac{\alpha_{2,0}(t)}{r^{2}}+\mathcal{O}\left(\frac{\ln r}{r^{3}}\right), \\
\chi=\frac{2 m_{f}}{r}+\frac{8 \dot{a} m_{f}}{a r^{2}}+\frac{1}{r^{3}}\left(\chi_{3,0}(t)-\left(\frac{32}{3} m_{f}^{3}+16 m_{f} \frac{\ddot{a}}{a}+16 m_{f} \frac{(\dot{a})^{2}}{a^{2}}\right) \ln r\right)+\mathcal{O}\left(\frac{\ln r}{r^{4}}\right), \\
\begin{array}{c}
A=\frac{r^{2}}{8}-\frac{\dot{a} r}{a}-\frac{2}{3} m_{f}^{2}+\frac{1}{r^{2}}\left(a_{2,0}(t)+\left(\frac{32}{9} m_{f}^{4}+\frac{16}{9} m_{b}^{2} \alpha_{2,0}(t)-\frac{32}{81} m_{b}^{4}+\frac{32 \ddot{a}}{3 a} m_{f}^{2}\right) \ln r\right. \\
\left.\quad-\frac{64}{27} m_{b}^{4} \ln ^{2} r\right)+\mathcal{O}\left(\frac{\ln ^{2} r}{r^{3}}\right),
\end{array}
\end{gathered}
$$

the observables of interest can be computed following the holographic renormalization of the model, most recently reviewed in [38],

$$
\begin{aligned}
\frac{32 \pi^{2}}{N^{2}} \mathcal{E}(t)= & -\frac{3}{8} a_{2,0}+3 \frac{(\dot{a})^{4}}{a^{4}}-\frac{1}{4} \chi_{3,0} m_{f}-\frac{1}{8} \alpha_{2,0}^{2}+\frac{5}{9} m_{b}^{2} \alpha_{2,0}-\left(\frac{8}{3} \ln 2+\frac{40}{81}\right) m_{b}^{4} \\
& +\left(\frac{8}{3} \ln 2+\frac{7}{9}\right) m_{f}^{4}+m_{f}^{2}\left(\frac{16 \ddot{a}}{3 a}+\frac{(\dot{a})^{2}}{a^{2}}(8 \ln 2+6)\right)+\mathcal{O}_{\mathcal{E}}^{\text {ambiguity }} \\
\frac{32 \pi^{2}}{N^{2}} P(t)= & -\frac{1}{8} a_{2,0}-\frac{4(\dot{a})^{2} \ddot{a}}{a^{3}}+\frac{(\dot{a})^{4}}{a^{4}}+\frac{1}{12} \chi_{3,0} m_{f}-\frac{1}{24} \alpha_{2,0}^{2}-\frac{13}{27} m_{b}^{2} \alpha_{2,0} \\
& +\left(\frac{8}{3} \ln 2+\frac{68}{243}\right) m_{b}^{4}+\left(\frac{7}{27}-\frac{8}{3} \ln 2\right) m_{f}^{4}-m_{f}^{2}\left(\left(\frac{16}{3} \ln 2+\frac{8}{9}\right) \frac{\ddot{a}}{a}\right. \\
& \left.+\left(\frac{8}{3} \ln 2+2\right) \frac{(\dot{a})^{2}}{a^{2}}\right)+\mathcal{O}_{P}^{\text {ambiguity }}
\end{aligned}
$$

\footnotetext{
${ }^{6}$ As explained in [36], the asymptotic expansion is determined up to a radial coordinate shifts $r \rightarrow r+\lambda(t)$, with an arbitrary function $\lambda(t)$. We fixed this residual reparametrization specifying the the $\mathcal{O}(r)$ term in the asymptotic expansion for the metric function $A$. We verified that the physical observables are independent of the reparametrization choice.
} 


$$
\begin{aligned}
\frac{32 \pi^{2}}{N^{2}} \mathcal{O}_{3}(t)= & -\frac{1}{4} \chi_{3,0}+\left(\frac{16}{3} \ln 2-\frac{2}{3}\right) m_{f}^{3}+m_{f}\left((8 \ln 2+2) \frac{\ddot{a}}{a}+(8 \ln 2+4) \frac{(\dot{a})^{2}}{a^{2}}\right) \\
& +\mathcal{O}_{3}^{\text {ambiguity }} \\
\frac{32 \pi^{2}}{N^{2}} \mathcal{O}_{2}(t)= & 2 \alpha_{2,0}-\frac{32}{3} m_{b}^{2} \ln 2+\mathcal{O}_{2}^{\text {ambiguity }},
\end{aligned}
$$

where $\mathcal{O}^{\text {ambiguity }}$ denote renormalization-scheme dependent ambiguities of the expectation values, parameterized by $\delta_{1}, \cdots \delta_{4},[38]$ :

$$
\begin{aligned}
\mathcal{O}_{\mathcal{E}}^{\text {ambiguity }} & =\frac{1}{4} m_{f}^{4} \delta_{1}+\frac{1}{9} m_{b}^{4} \delta_{4}+\frac{(\dot{a})^{2}}{a^{2}}\left(\delta_{2} m_{b}^{2}+\frac{3}{2} \delta_{3} m_{f}^{2}\right), \\
\mathcal{O}_{P}^{\text {ambiguity }} & =-\frac{1}{4} m_{f}^{4} \delta_{1}-\frac{1}{9} m_{b}^{4} \delta_{4}-\left(\frac{2 \ddot{a}}{a}+\frac{(\dot{a})^{2}}{a^{2}}\right)\left(\frac{1}{3} \delta_{2} m_{b}^{2}+\frac{1}{2} \delta_{3} m_{f}^{2}\right), \\
\mathcal{O}_{3}^{\text {ambiguity }} & =\frac{1}{2} \delta_{1} m_{f}^{3}+\frac{3}{2} m_{f} \delta_{3}\left(\frac{\ddot{a}}{a}+\frac{(\dot{a})^{2}}{a^{2}}\right), \\
\mathcal{O}_{2}^{\text {ambiguity }} & =\frac{4}{9} \delta_{4} m_{b}^{2}+2 \delta_{2}\left(\frac{\ddot{a}}{a}+\frac{(\dot{a})^{2}}{a^{2}}\right) .
\end{aligned}
$$

Independent of the regularization scheme parameters $\delta_{1}, \cdots, \delta_{4}$, these expectation values satisfy the expected conformal anomaly relation

$$
-\mathcal{E}+3 P=\frac{N^{2}}{32 \pi^{2}}\left(R_{\mu \nu} R^{\mu \nu}-\frac{1}{3} R^{2}\right)-2 m_{f} \mathcal{O}_{3}-m_{b}^{2} \mathcal{O}_{2}-\frac{N^{2}}{24 \pi^{2}}\left(m_{f}^{4}-m_{b}^{4}+\frac{m_{f}^{2}}{2} R\right),
$$

Furthermore, the conservation of the stress-energy tensor

$$
\frac{d \mathcal{E}}{d t}+3 \frac{\dot{a}}{a}(\mathcal{E}+P)=0
$$

is a consequence of the momentum constraint (3.4):

$$
\begin{aligned}
0= & \dot{a}_{2,0}+\frac{4 \dot{a} a_{2,0}}{a}+\frac{2}{3} \alpha_{2,0} \dot{\alpha}_{2,0}+\frac{4 \dot{a} \alpha_{2,0}^{2}}{3 a}+\left(\frac{416}{243} m_{b}^{4}-\frac{224}{27} m_{f}^{4}\right) \frac{\dot{a}}{a} \\
& -\left(\frac{160 \dot{a} \ddot{a}}{3 a^{2}}+\frac{128 \dddot{a}}{9 a}\right) m_{f}^{2}-\left(\frac{16 \dot{a} \alpha_{2,0}}{27 a}+\frac{40 \alpha_{2,0}}{27}\right) m_{b}^{2}+\left(\frac{4 \dot{a} \chi_{3,0}}{3 a}+\frac{2 \dot{\chi}_{3,0}}{3}\right) m_{f} .
\end{aligned}
$$

One of the advantages of the holographic formulation of a QFT dynamics is the natural definition of its far-from-equilibrium entropy density. A gravitational geometry (3.1) has an apparent horizon located at $r=r_{\mathrm{AH}}$, where [36]

$$
\left.d_{+} \Sigma\right|_{r=r_{\mathrm{AH}}}=0
$$

Following $[39,40]$ we associate the non-equilibrium entropy density $s$ of the boundary QFT with the Bekenstein-Hawking entropy density of the apparent horizon

$$
a^{3} s=\left.\frac{\Sigma^{3}}{4 G_{5}}\right|_{r=r_{\mathrm{AH}}}=\left.\frac{N^{2} \Sigma^{3}}{16 \pi}\right|_{r=r_{\mathrm{AH}}}
$$


with $a$ being the QFT background geometry scale factor (1.5). Using the holographic background equations of motion (3.2)-(3.4) we find [29]

$$
\frac{d\left(a^{3} s\right)}{d t}=\left.\frac{2 N^{2}}{\pi}\left(\Sigma^{3}\right)^{\prime} \frac{\left.\left(d_{+} \chi\right)^{2}+3\left(d_{+} \alpha\right)^{2}\right)}{-4 \mathcal{P}}\right|_{r=r_{\mathrm{AH}}} .
$$

It was shown in [29] that (3.17) correctly reproduces the entropy growth in the hydrodynamic regime, due to the bulk viscosity of the gauge theory plasma. In appendix A we prove that the entropy production rate as defined by (3.17) is non-negative, i.e.,

$$
\frac{d\left(a^{3} s\right)}{d t} \geq 0
$$

in holographic dynamics governed by (3.2)-(3.4),

Dynamical evolution of homogeneous and isotropic states of $\mathcal{N}=2^{*}$ gauge theory in $d S_{4}$ within holographic framework proceeds as follows:

- First, we define parameters of the theory providing the mass parameters $\left\{m_{b}, m_{f}\right\}$ and the Hubble constant $H$.

- An initial state is specified providing gravitational scalar profiles

$$
\alpha(t=0, r)=\alpha_{\text {initial }}(r), \quad \chi(t=0, r)=\chi_{\text {initial }}(r),
$$

subject to the boundary conditions (3.5). The Hamiltonian constraint (3.3) and the last two evolution equations in (3.2) subject to the boundary conditions (3.5) along with

$$
d_{+} \alpha=\frac{2 m_{b}^{2} \ln r}{3 r}+\mathcal{O}\left(r^{-1}\right), \quad d_{+} \chi=-\frac{m_{f}}{4}+\mathcal{O}\left(r^{-1}\right),
$$

are solved to determine $\Sigma_{\text {initial }}$ and $\{\alpha, \chi\}_{\text {initial }}$,

$$
\Sigma_{\text {initial }}(r) \equiv \Sigma(t=0, r), \quad\{\alpha, \chi\}_{\text {initial }}(r) \equiv\{\alpha, \chi\}(t=0, r) .
$$

Initial state specification is completed with solving for $A_{\text {initial }}(r) \equiv A(t=0, r)$ (the second equation in (3.2)) subject to the boundary condition (3.6) with $\alpha_{2,0}(t=0)$ extracted from $\alpha_{\text {initial }}$ and specifying a free parameter $a_{2,0}^{\text {initial }}, a_{2,0}(t=0)=a_{2,0}^{\text {initial }}$. The latter parameter determines the initial energy density of the state, following (3.7).

- This initial state can be evolved following [36]. At any time $t$ we can compute the one point correlation functions (3.7)-(3.10) and the entropy density of the state (3.16). It is also possible to compute additional non-local observables of the evolution, for example as in [41].

Implementing the holographic evolution as explained above is technically challenging, and is beyond the scope of this paper. The difficulty ${ }^{7}$ is not intrinsic to a $d S_{4}$ background metric for the boundary gauge theory - in fact, we prove in appendix B the precise map

\footnotetext{
${ }^{7}$ It is related to the presence of log-terms in the near-boundary expansion for the dual gravitational scalars (3.6). We hope to solve this problem in the future.
} 
between the holographic evolution of the $\mathcal{N}=2^{*}$ states in de Sitter and in Minkowski space-times:

\begin{tabular}{|c|c|c|}
\hline & $d S_{4}$ & $R^{3,1}$ \\
\hline background metric & $d s^{2}=-d t^{2}+e^{2 H t} d \boldsymbol{x}^{2}$ & $d \hat{s}^{2}=-d \tau^{2}+d \boldsymbol{x}^{2}$ \\
\hline evolution time & $t \in[0, \infty)$ & $\tau=\left(1-e^{-H t}\right) / H \quad \in\left[0, \frac{1}{H}\right)$ \\
\hline mass parameters & $\left\{m_{b}, m_{f}\right\}$ & $\left\{\hat{m}_{b}(\tau)=\frac{m_{b}}{1-H \tau}, \hat{m}_{f}(\tau)=\frac{m_{f}}{1-H \tau}\right\}$ \\
\hline
\end{tabular}

\section{Vacuum of $\mathcal{N}=2^{*}$ gauge theory in $d S_{4}$}

In the previous section we outlined the setup for the holographic evolution of a generic homogeneous and isotropic state of $\mathcal{N}=2^{*}$ gauge theory. While we have not implemented the evolution, it is physically reasonable to expect that any initial, finite energy density state of the theory in de Sitter background would evolve as $t \rightarrow \infty$ into a universal static state, which we label as vacuum. Indeed, one expects that energy density $\mathcal{E}(t)$ is diluted according to (3.13), until it reaches the (constant) vacuum value $\mathcal{E}_{v}$, entirely determined by the Hubble constant $H$ and the relevant microscopic couplings of the theory $\left\{m_{b}, m_{f}\right\}$, i.e., $\mathcal{E}_{v}=\mathcal{E}_{v}\left(H, m_{b}, m_{f}\right)$ :

$$
\lim _{t \rightarrow \infty} \mathcal{E}(t)=-\lim _{t \rightarrow \infty} P(t)=\mathcal{E}_{v}, \quad \lim _{t \rightarrow \infty} \dot{\mathcal{E}}(t)=0 .
$$

From (3.7) we then expect that the normalizable coefficients of the gravitational bulk dynamics $\left\{a_{2,0}, \chi_{3,0}, \alpha_{2,0}\right\}$ approach constant values at late times:

$$
\begin{aligned}
\lim _{t \rightarrow \infty}\left\{a_{2,0}, \chi_{3,0}, \alpha_{2,0}\right\}(t) & =\left\{a_{2,0}, \chi_{3,0}, \alpha_{2,0}\right\}^{v}, \\
\lim _{t \rightarrow \infty}\left\{\dot{a}_{2,0}, \dot{\chi}_{3,0}, \dot{\alpha}_{2,0}\right\}(t) & =\{0,0,0\} .
\end{aligned}
$$

Note that $a_{2,0}^{v}$ is not independent from $\left\{\chi_{3,0}, \alpha_{2,0}\right\}^{v}$; from (3.14):

$$
a_{2,0}^{v}=\frac{152}{9} H^{2} m_{f}^{2}-\frac{104}{243} m_{b}^{4}+\frac{56}{27} m_{f}^{4}+\frac{4}{27} \alpha_{2,0}^{v} m_{b}^{2}-\frac{1}{3} \chi_{3,0}^{v} m_{f}-\frac{1}{3}\left(\alpha_{2,0}^{v}\right)^{2} .
$$

Given (4.2), it is straightforward to see that to any order in the boundary expansion $\{\alpha, \chi, A\}$ become static at late times:

$$
\lim _{t \rightarrow \infty}\{\alpha, \chi, A\}(t, r)=\{\alpha, \chi, A\}_{v}(r)
$$

Finally, (3.3) along with the boundary condition (3.5) implies that $\frac{\Sigma}{a}$ becomes static at late times:

$$
\lim _{t \rightarrow \infty} \frac{\Sigma(t, r)}{a(t)}=\sigma_{v}(r) .
$$

Dropping the time dependence according to (4.4) and (4.5) we obtain from (3.2)-(3.4) the following set of dual gravitational equations describing the vacuum of $\mathcal{N}=2^{*}$ gauge 
theory in $d S_{4}$ :

$$
\begin{aligned}
0 & =\alpha_{v}^{\prime \prime}+\left(\frac{3 H}{2 A_{v}}+\left(\ln A_{v} \sigma_{v}^{3}\right)^{\prime}\right) \alpha_{v}^{\prime}-\frac{1}{48 A_{v}} \partial_{\alpha} \mathcal{P}, \\
0 & =\chi_{v}^{\prime \prime}+\left(\frac{3 H}{2 A_{v}}+\left(\ln A_{v} \sigma_{v}^{3}\right)^{\prime}\right) \chi_{v}^{\prime}-\frac{1}{16 A_{v}} \partial_{\chi} \mathcal{P}, \\
0 & =\sigma_{v}^{\prime \prime}+\frac{4}{3} \sigma_{v}\left(3\left(\alpha_{v}^{\prime}\right)^{2}+\left(\chi_{v}^{\prime}\right)^{2}\right), \\
0 & =A_{v}^{\prime \prime}+4 A_{v}^{\prime}\left(3\left(\alpha_{v}^{\prime}\right)^{2}+\left(\chi_{v}^{\prime}\right)^{2}\right)-6 A_{v}\left(\left(\ln \sigma_{v}\right)^{\prime}\right)^{2}-6 H\left(\ln \sigma_{v}\right)^{\prime}-\frac{\mathcal{P}}{6},
\end{aligned}
$$

along with the constraints

$$
\begin{aligned}
& 0=\sigma_{v}^{\prime}+\frac{\sigma_{v}}{2 A_{v}}\left(H-A_{v}^{\prime}\right), \\
& 0=\left(\alpha_{v}^{\prime}\right)^{2}+\frac{1}{3}\left(\chi_{v}^{\prime}\right)^{2}-\frac{1}{2}\left(\left(\ln \sigma_{v}\right)^{\prime}\right)^{2}-\frac{\sigma_{v}^{\prime}}{4 \sigma_{v} A_{v}}\left(2 H+A_{v}^{\prime}\right)+\frac{H}{8 A_{v}^{2}}\left(H-A_{v}^{\prime}\right)-\frac{\mathcal{P}}{24 A_{v}} .
\end{aligned}
$$

It is straightforward to verify that constraints (4.7) are consistent with (4.6).

Vacuum solution has to satisfy the boundary condition (see (3.5))

$$
\begin{aligned}
\sigma_{v} & =\frac{r+\lambda}{2}+\mathcal{O}\left(r^{-1}\right), & A_{v} & =\frac{(r+\lambda)^{2}}{8}-H(r+\lambda)+\mathcal{O}\left(r^{0}\right), \\
\alpha_{v} & =-\frac{8 m_{b}^{2} \ln r}{3 r^{2}}+\mathcal{O}\left(r^{-2}\right), & \chi_{v} & =\frac{2 m_{f}}{r}+\mathcal{O}\left(r^{-2}\right),
\end{aligned}
$$

where we reintroduced time-independent radial coordinate shift symmetry $r \rightarrow r+\lambda$, see [36]. It is important to realize that the boundary condition (4.8) is not sufficient to identify a unique solution. Note that at late times the apparent horizon settles at a fixed radial location $r_{\mathrm{AH}}$ determined from

$$
d_{+} \Sigma\left(t, r_{\mathrm{AH}}\right)=0 \quad \Longleftrightarrow \quad H \sigma_{v}+\left.A_{v} \sigma_{v}^{\prime}\right|_{r=r_{\mathrm{AH}}}=0 .
$$

We need to construct a solution to (4.6)-(4.7) for $r \in\left(r_{\mathrm{AH}}, \infty\right)$. As shown in appendix A, $\sigma_{v}^{\prime}>0$ for $r>r_{\mathrm{AH}}$; additionally, the absence of naked singularities in the gravitational dual requires that $\sigma_{v}>0$ for $r>r_{\mathrm{AH}}$ - thus, (4.9) implies that $A_{v}\left(r_{\mathrm{AH}}\right)<0$. Since $A_{v} \rightarrow \infty$ as $r \rightarrow \infty$, there must be a radial location $r=r_{s}>r_{\mathrm{AH}}$, such that $A_{v}\left(r_{s}\right)=0$. Inspection of the bulk equations of motion (4.6)-(4.7) shows that they are generically singular when $A_{v}=0$. Avoiding this singularity provides complementary conditions to uniquely identify the $d S_{4}$ vacuum of $\mathcal{N}=2^{*}$ gauge theory.

The rest of this section is organized as follows. We prove that the $d S_{4}$ vacuum of $\mathcal{N}=2^{*}$ gauge theory obtained as a late-time attractor of the evolution from a homogeneous and isotropic initial state is a Bunch-Davies vacuum. We explicitly compute the vacuum energy of the theory $\mathcal{E}_{v}$ for $\left\{m_{f}=0, m_{b} \neq 0\right\}$ and in a supersymmetric case $m_{f}=m_{b}$. Following (3.17), we show that non-conformal gauge theory vacuum is not an adiabatic state - the rate of the comoving entropy production is non-zero:

$$
\lim _{t \rightarrow \infty} \frac{1}{H^{3} a^{3}} \frac{d}{d t}\left(a^{3} s\right) \equiv 3 H \times \mathcal{R}=\text { const } \neq 0 .
$$

To contrast dynamics of non-conformal gauge theories with conformal ones, we review $\mathcal{N}=4 \mathrm{SYM}$ in $d S_{4}$ in appendix $\mathrm{C}$. 


\section{1 $d S_{4}$ vacuum is Euclidean}

Bunch-Davies (Euclidean) vacuum of $\mathcal{N}=2^{*}$ gauge theory was constructed in [9]. We review the main points of the construction and prove that the holographic vacuum equations (4.6) and (4.7) are equivalent to those in [9].

To describe Bunch-Davies vacuum of $\mathcal{N}=2^{*}$ gauge theory at strong coupling one studies the effective gravitational action (2.5) within the following background ansatz:

$$
d s_{5}^{2}=-c_{1}^{2}\left(d \mathcal{M}_{4}\right)^{2}+c_{2}^{2}(d z)^{2}, \quad c_{i}=c_{i}(z), \quad \chi=\chi(z), \quad \alpha=\alpha(z)
$$

where $\left(d \mathcal{M}_{4}\right)^{2}$ is the de Sitter boundary gauge theory metric. Irrespectively whether this metric is written in flat or closed slicing $\left(d \mathcal{M}_{4}^{f / c}\right)^{2}$,

$$
\left(d \mathcal{M}_{4}^{f}\right)^{2}=-d t^{2}+e^{2 H t} d \boldsymbol{x}^{2}, \quad\left(d \mathcal{M}_{4}^{c}\right)^{2}=-d t^{2}+\frac{1}{H^{2}} \cosh ^{2}(H t)\left(d S^{3}\right)^{2},
$$

the dual gravitational equations of motion take form

$$
\begin{aligned}
& 0=\alpha^{\prime \prime}+\left(\ln \frac{c_{1}^{4}}{c_{2}}\right)^{\prime} \alpha^{\prime}-\frac{c_{2}^{2}}{24} \partial_{\alpha} \mathcal{P}, \\
& 0=\chi^{\prime \prime}+\left(\ln \frac{c_{1}^{4}}{c_{2}}\right)^{\prime} \chi^{\prime}-\frac{c_{2}^{2}}{8} \partial_{\chi} \mathcal{P}, \\
& 0=c_{1}^{\prime \prime}+\frac{\left(c_{1}^{\prime}\right)^{2}}{c_{1}}-\frac{c_{1}^{\prime} c_{2}^{\prime}}{c_{2}}-\frac{c_{2}^{2} H^{2}}{c_{1}}+2 c_{1}\left(\left(\alpha^{\prime}\right)^{2}+\frac{1}{3}\left(\chi^{\prime}\right)^{2}\right)+\frac{1}{6} c_{1} c_{2}^{2} \mathcal{P}, \\
& 0=\left(\alpha^{\prime}\right)^{2}+\frac{1}{3}\left(\chi^{\prime}\right)^{2}-\frac{\left(c_{1}^{\prime}\right)^{2}}{c_{1}^{2}}+\frac{c_{2}^{2} H^{2}}{c_{1}^{2}}-\frac{1}{12} c_{2}^{2} \mathcal{P} .
\end{aligned}
$$

Notice that closed slicing de Sitter metric makes Euclidean analytic continuation obvious:

$$
\left.\left(d \mathcal{M}_{4}^{c}\right)^{2}\right|_{t \rightarrow i t \equiv t_{E} \equiv \frac{\theta}{H}} \rightarrow \frac{1}{H^{2}}\left(d \theta^{2}+\cos ^{2} \theta\left(d S^{3}\right)^{2}\right)=\frac{1}{H^{2}}\left(d S^{4}\right)^{2} .
$$

It is straightforward to relate (4.13) and (4.6), (4.7): assuming that as $z \rightarrow \infty$ the warp factor $c_{1} \rightarrow \infty$, the systems of equations become identical with

$$
\begin{aligned}
& r=-\int_{z}^{\infty} d s c_{1}(s) c_{2}(s), \quad \sigma_{v}(r)=c_{1}(z) \exp \left[H \int_{z}^{\infty} d s \frac{c_{2}(s)}{c_{1}(s)}\right], \\
& A_{v}(r)=\frac{c_{1}(z)^{2}}{2}, \quad \alpha_{v}(r)=\ln \rho(z), \quad \chi_{v}(r)=\chi(z) .
\end{aligned}
$$

Of course, the map (4.15) is nothing but the map between the asymptotic $t \rightarrow \infty$ infalling Eddington-Finkelstein metric (3.1) and the Fefferman-Graham metric (4.11). The advantage to use the characteristic formulation in the dual gravitational description (3.1) is that it clarifies the selection of the Bunch-Davies vacuum for the strongly coupled gauge theory. As we will see shortly, it also explains why the Bunch-Davies vacuum is non-adiabatic. 


\section{$4.2 \mathcal{E}_{v}$ of $\mathcal{N}=2^{*}$ gauge theory in $d S_{4}$}

In this section we numerically solve the equations of motion (4.6), (4.7) and compute $\mathcal{E}_{v}$ for two representative holographic RG flows: $m_{f}=m_{b}$ and $\left\{m_{f}=0, m_{b} \neq 0\right\}$.

Introducing a new radial coordinate

$$
x=\frac{H}{r},
$$

and redefining

$$
A_{v} \equiv \frac{H^{2}}{8 x^{2}} a_{0}(x), \quad \sigma_{v} \equiv \frac{H}{2 x} s_{0}(x), \quad \alpha_{v} \equiv \ln \left(r_{0}(x)\right), \quad \cosh \chi_{v} \equiv c_{0}(x),
$$

from (4.6) and (4.7) we find:

$$
\begin{aligned}
& 0=r_{0}^{\prime \prime}+\frac{2 r_{0}^{\prime}}{x}-\frac{\left(r_{0}^{\prime}\right)^{2}}{r_{0}}-\frac{5 r_{0}^{\prime} \Theta}{3 a_{0} x r_{0}^{2}}-\frac{2}{3} \frac{2 c_{0}^{2} r_{0}^{12}\left(c_{0}^{2}-1\right)-r_{0}^{6}\left(2 c_{0}^{2}-1\right)+1}{x^{2} r_{0}^{3} a_{0}}, \\
& 0=c_{0}^{\prime \prime}+\frac{2 c_{0}^{\prime}}{x}-\frac{c_{0}\left(c_{0}^{\prime}\right)^{2}}{c_{0}^{2}-1}-\frac{5 c_{0}^{\prime} \Theta}{3 a_{0} x r_{0}^{2}}-\left(c_{0}^{2}-1\right) \frac{\left(\left(2 c_{0}^{2}-1\right) r_{0}^{6}-4\right) r_{0}^{2} c_{0}}{a_{0} x^{2}}, \\
& 0=a_{0}^{\prime}-\frac{2 a_{0}}{x}+\frac{2 \Theta}{3 r_{0}^{2} x} \\
& 0=\frac{s_{0}^{\prime}}{s_{0}}-\frac{1}{x}-\frac{4}{a_{0}}+\frac{\Theta}{3 a_{0} x r_{0}^{2}}
\end{aligned}
$$

where

$$
\Theta^{2}=9\left(r_{0}^{\prime}\right)^{2} r_{0}^{2} a_{0}^{2} x^{2}+\frac{3 a_{0}^{2} x^{2} r_{0}^{4}\left(c_{0}^{\prime}\right)^{2}}{c_{0}^{2}-1}+\left(3 c_{0}^{2} r_{0}^{12}\left(1-c_{0}^{2}\right)+6 r_{0}^{6}\left(2 c_{0}^{2}-1\right)+3\right) a_{0}+144 x^{2} r_{0}^{4} .
$$

Equations (4.18)-(4.21) have the following boundary asymptotics (we keep only the couple relevant terms)

$$
\begin{aligned}
& c_{0}=1+x^{2} c_{2,0}-x^{3} c_{2,0} a_{1,0}+x^{4}\left(\left(\frac{16}{3} c_{2,0}^{2}+32 c_{2,0}\right) \ln x+c_{4,0}\right)+\mathcal{O}\left(x^{5} \ln x\right), \\
& r_{0}=1+x^{2}\left(r_{2,1} \ln x+r_{2,0}\right)+\mathcal{O}\left(x^{3} \ln x\right), \quad a_{0}=1+a_{1,0} x+\mathcal{O}\left(x^{2}\right), \\
& s_{0}=1+\left(4+\frac{a_{1,0}}{2}\right) x+\mathcal{O}\left(x^{2}\right),
\end{aligned}
$$

where the non-normalizable coefficients $\left\{r_{2,1}, c_{2,0}\right\}$ and the gauge parameter $a_{1,0}$ are related to $\left\{m_{b}, m_{f}\right\}$ and $\lambda$ in (4.8) as follows

$$
r_{2,1}=\frac{8 m_{b}^{2}}{3 H^{2}}, \quad c_{2,0}=\frac{2 m_{f}^{2}}{H^{2}}, \quad a_{1,0}=\frac{8 \lambda}{H} .
$$

The normalizable coefficients $\left\{r_{2,0}, c_{4,0}\right\}$ determine expectation values $\left\{\mathcal{O}_{2}^{v}, \mathcal{O}_{3}^{v}\right\}$ and the vacuum energy density $\mathcal{E}_{v}$. Specifically,

$$
\begin{aligned}
\frac{32 \pi^{2}}{N^{2}} \mathcal{E}_{v}= & 3 H^{4}+\frac{r_{2,0}}{2} m_{b}^{2} H^{2}-\frac{c_{4,0}}{16} H^{4}+\frac{3\left(a_{1,0}\right)^{2}}{32} m_{f}^{2} H^{2} \\
& +\left(\frac{4}{3}\left(m_{b}^{4}-m_{f}^{4}\right)-4 m_{f}^{2} H^{2}\right) \ln \frac{H}{\Lambda}+q_{1} m_{f}^{2} H^{2}+q_{2} m_{f}^{4}+q_{3} m_{b}^{2} H^{2}+q_{4} m_{b}^{4},
\end{aligned}
$$


where $\Lambda$ and $q_{i}$ are arbitrary renormalization scheme choice parameters, related to $\delta_{i}$ in (3.11). The ambiguities of the vacuum energy density discussed here mirror the wellknown ambiguities defining the stress-energy tensor of weakly coupled QFTs in curved space-times [5]. In section 5 we discuss the implication of these ambiguities in the context of self-consistent inflationary scenarios (1.6).

As we already mentioned, the choice of the gauge parameter $\lambda$ (correspondingly $a_{1,0}$ in (4.23)) is completely arbitrary. We find it convenient to adjust $a_{1,0}=a_{1,0}\left(r_{2,1}, c_{2,0}\right)$ in such a way that the zero of the warp function $a_{0}$ occurs at exactly the same location as it is for the $\mathcal{N}=4 \mathrm{SYM}$ duality where $r_{2,1}=c_{2,0}=0$ (see (C.7)):

$$
\left.a_{0}(x)\right|_{x=x_{s}=\frac{1}{9}}=0,
$$

implying that the range of $x$ is

$$
x \in\left(0, \frac{1}{9}\right] .
$$

The absence of singularities in (4.18)-(4.21) as $y \equiv\left(\frac{1}{9}-x\right) \rightarrow 0_{+}$enforces the following asymptotic behaviour

$$
\begin{aligned}
& a_{0}=8 y+\left(\frac{27}{r_{0, s, 0}^{4}}-27 r_{0, s, 0}^{2} c_{0, s, 0}^{2}\left(c_{0, s, 0}^{2} r_{0, s, 0}^{6}-r_{0, s, 0}^{6}-4\right)-54 r_{0, s, 0}^{2}-72\right) y^{2}+\mathcal{O}\left(y^{3}\right), \\
& s_{0}=s_{0, s, 0}+\mathcal{O}(y), \quad r_{0}=r_{0, s, 0}+\mathcal{O}(y), \quad c_{0}=c_{0, s, 0}+\mathcal{O}(y)
\end{aligned}
$$

determined by 3 parameters $\left\{r_{0, s, 0}, c_{0, s, 0}, s_{0, s, 0}\right\}$. The total order of the system of the differential equations (4.18)-(4.21) is 6 ; thus, along with the normalizable coefficients $\left\{r_{2,0}, c_{4,0}\right\}$ and the gauge parameter $a_{1,0}$, we have the correct number of the adjustable coefficients necessary to uniquely identify the gravitational solution for a given set of $\left\{\frac{m_{b}}{H}, \frac{m_{f}}{H}\right\}$.

In the limit $\left\{r_{2,1}, c_{2,0}\right\} \ll 1(4.18)-(4.21)$ can be solved analytically. To order $\mathcal{O}\left(r_{2,1}^{2}, c_{2,0}\right), a_{0}$ and $s_{0}$ are that of the $\mathcal{N}=4 \mathrm{SYM}$, discussed in appendix $\mathrm{C}$, while

$$
\begin{aligned}
r_{0}= & 1-r_{2,1} x^{2}\left(\frac{5 x-1}{2} \ln \frac{\sqrt{(1-9 x)(1-x)}+5 x-1}{5 x-1-\sqrt{(1-9 x)(1-x)}}-\sqrt{(1-9 x)(1-x)}\right) \\
& \times \frac{1}{(1-9 x)^{3 / 2}(1-x)^{3 / 2}}+\mathcal{O}\left(r_{2,1}^{2}, c_{2,0}^{2}\right) \\
c_{0}= & 1+c_{2,0} x^{2}\left(8 x^{2} \ln \frac{\sqrt{(1-9 x)(1-x)}+5 x-1}{5 x-1-\sqrt{(1-9 x)(1-x)}}-(5 x-1) \sqrt{(1-9 x)(1-x)}\right)^{2} \\
& \times \frac{1}{(1-9 x)^{3}(1-x)^{3}}+\mathcal{O}\left(r_{2,1}^{2}, c_{2,0}^{2}\right) .
\end{aligned}
$$

For general values of $\left\{r_{2,1}, c_{2,0}\right\}$ the system (4.18)-(4.21) is solved numerically, using the shooting method introduced in [42].

Figure 1 presents characteristic profiles of the gravitational scalar $\left\{r_{0}, c_{0}\right\}$ for select (small) values of $\left\{m_{b}, m_{f}\right\}$. For comparison, we added perturbative predictions given by (4.29) and (4.30). 

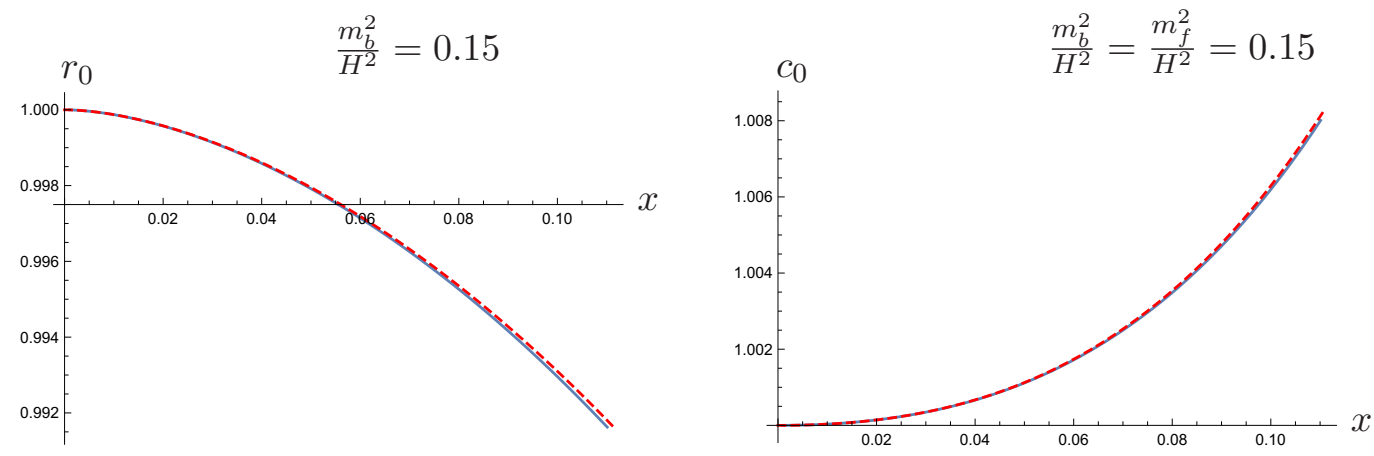

Figure 1. Profiles of the gravitational scalars $\left\{r_{0}, c_{0}\right\}$ (solid curves) describing the holographic dual to $\mathcal{N}=2^{*}$ gauge theory vacuum in $d S_{4}$ for select values of $\left\{m_{b}, m_{f}\right\}$. The dashed red lines represent the analytic solutions valid for $\left\{m_{b}, m_{f}\right\} \ll H$, see (4.29) and (4.30).
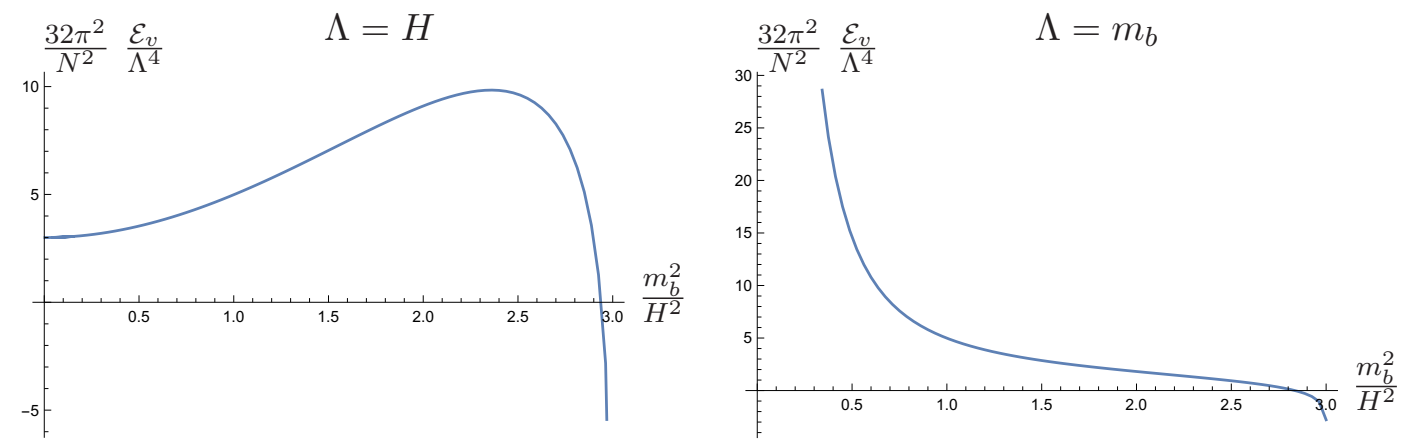

Figure 2. Vacuum energy $\mathcal{E}_{v}($ see $(4.25))$ of $\mathcal{N}=2^{*}$ gauge theory in $d S_{4}$ with $\left\{m_{b} \neq 0, m_{f}=0\right\}$. We choose renormalization parameters $q_{i}=0$, and set the renormalization scale $\Lambda=H$ (left panel) and $\Lambda=m_{b}$ (right panel).

Having obtained numerical solutions to (4.18)-(4.21), we extract the normalizable coefficients $\left\{r_{2,0}, c_{4,0}\right\}$. We compute the vacuum energy density following (4.25). The results are renormalization scheme dependent, and we set $q_{i}=0$. There is a remaining ambiguity associated with the choice of the renormalization scale $\Lambda$. This ambiguity is important, because of the interesting $\ln \frac{H}{\Lambda}$ dependence in the expression for the vacuum energy. We consider setting $\Lambda=H$ and $\Lambda=m_{b}$. In the former case the log-dependence disappears, and one can take the limit $\frac{m_{b}}{H} \rightarrow 0$, reproducing the $\mathcal{N}=4$ SYM result (C.8). Results for the vacuum energy $\mathcal{E}_{v}$ of $\mathcal{N}=2^{*}$ gauge theory in $d S_{4}$ as a function of $\frac{m_{b}^{2}}{H^{2}}$ are collected in figures 2-3. Modulo the scheme dependence, we find that $\frac{\mathcal{E}_{v}}{\Lambda^{4}}>0$ for sufficiently small $\frac{m_{b}^{2}}{H^{2}}$; however, it becomes negative for large mass parameters $\left\{m_{b}, m_{f}\right\}$.

\section{3 $d S_{4}$ vacuum of a non-conformal gauge theory is non-adiabatic}

In appendix $\mathrm{C}$ we argued that the comoving entropy of $\mathcal{N}=4 \mathrm{SYM}$ vacuum in $d S_{4}$ is constant - the vacuum is an adiabatic state. Here, we compute the comoving entropy production rate $\mathcal{R}$ (see (4.10)) of $\mathcal{N}=2^{*}$ gauge theory and show that it is non-zero. Thus, we are led to claim that $d S_{4}$ vacua of non-conformal gauge theories are non-adiabatic. 

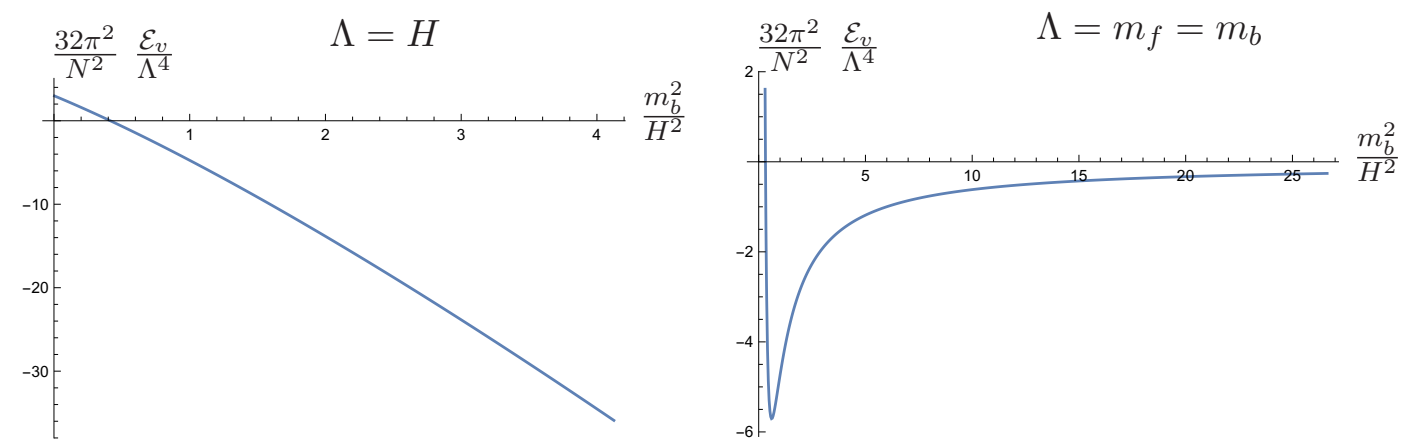

Figure 3. Vacuum energy $\mathcal{E}_{v}$ (see $\left.(4.25)\right)$ of $\mathcal{N}=2^{*}$ gauge theory in $d S_{4}$ with $\left\{m_{b}=m_{f}\right\}$. We choose renormalization parameters $q_{i}=0$, and set the renormalization scale $\Lambda=H$ (left panel) and $\Lambda=m_{b}$ (right panel).

As established in (4.9), the apparent horizon of the vacuum state of the $\mathcal{N}=2^{*}$ gauge theory in $d S_{4}$ occurs past the point along the radial holographic direction where $a_{v}$ vanishes. In the radial coordinate (4.16) this means that $x_{\mathrm{AH}}>x_{s}$. So we need to extend solution of (4.18)-(4.21) past $x=x_{s}$. The extension is simple for $\left\{r_{2,1}, c_{2,0}\right\} \ll 1$. Recall that to order $\mathcal{O}\left(r_{2,1}^{2}, c_{2,0}\right)$ the warp factors $a_{0}$ and $s_{0}$ are that of the $\mathcal{N}=4 \mathrm{SYM}$ represented by (C.6) - there is nothing particular about $x=x_{s}=\frac{1}{9}$ point. Analytic continuation of (4.29) and (4.30) for $x>x_{s}$ yields:

$$
\begin{gathered}
r_{0}=1-r_{2,1}\left(x^{2}\left((5 x-1) \arctan \frac{5 x-1}{\sqrt{(9 x-1)(1-x)}}+\sqrt{(9 x-1)(1-x)}\right)\right. \\
\left.\quad+\frac{1}{2} \pi x^{2}(5 x-1)\right) \frac{1}{(9 x-1)^{3 / 2}(1-x)^{3 / 2}}+\mathcal{O}\left(r_{2,1}^{2}, c_{2,0}^{2}\right), \\
c_{0}=1+c_{2,0} x^{2}\left(16 \arctan \frac{5 x-1}{\sqrt{(9 x-1)(1-x)}} x^{2}+(5 x-1) \sqrt{(9 x-1)(1-x)}\right. \\
\left.+8 \pi x^{2}\right)^{2} \frac{1}{(9 x-1)^{3}(1-x)^{3}}+\mathcal{O}\left(r_{2,1}^{2}, c_{2,0}^{2}\right) .
\end{gathered}
$$

For general values of $\left\{r_{2,1}, c_{2,0}\right\}$ we need to resort to numerics. Instead of solving (4.18)(4.19), we solve equations (4.6) (rewritten in $x$ coordinate), using as initial condition the asymptotic behaviour (4.28). In this process we do not solve the constraint equations (4.7), but rather monitor their residuals as a test on our analysis. These residuals are shown in figures $4-5$.

Figure 6 represents profiles of the gravitational scalars $\left\{r_{0}, c_{0}\right\}$ for $x>x_{s}$, for the same values of mass parameters as in figure 1. Notice that the deviation of the numerical solutions away from the perturbative results (4.31) and (4.32) becomes more pronounced as one approaches the apparent horizon, indicated by vertical green lines, i.e., the gravitational scalar fields backreaction is strong near the apparent horizon. In general, the apparent horizon is determined by locating the first zero in $d_{+} \Sigma$ for $x>x_{s}$, see (4.9). We illustrate the procedure in the left panel of figure 7 for $\mathcal{N}=2^{*}$ gauge theory vacuum in $d S_{4}$ at 


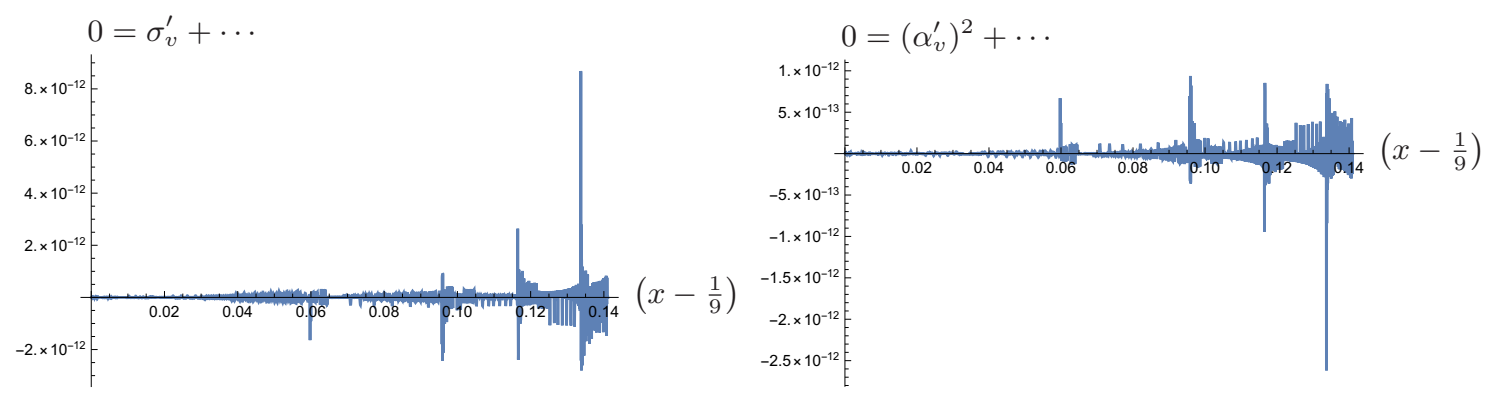

Figure 4. Constraint equations (4.6) are not integrated in constructing the gravitational background dual to vacuum of $\mathcal{N}=2^{*}$ gauge theory in the vicinity of the apparent horizon, i.e., for $x>x_{s}$. Rather, their residuals are used as a numerical test. Here $m_{b}=H$ and $m_{f}=0$.
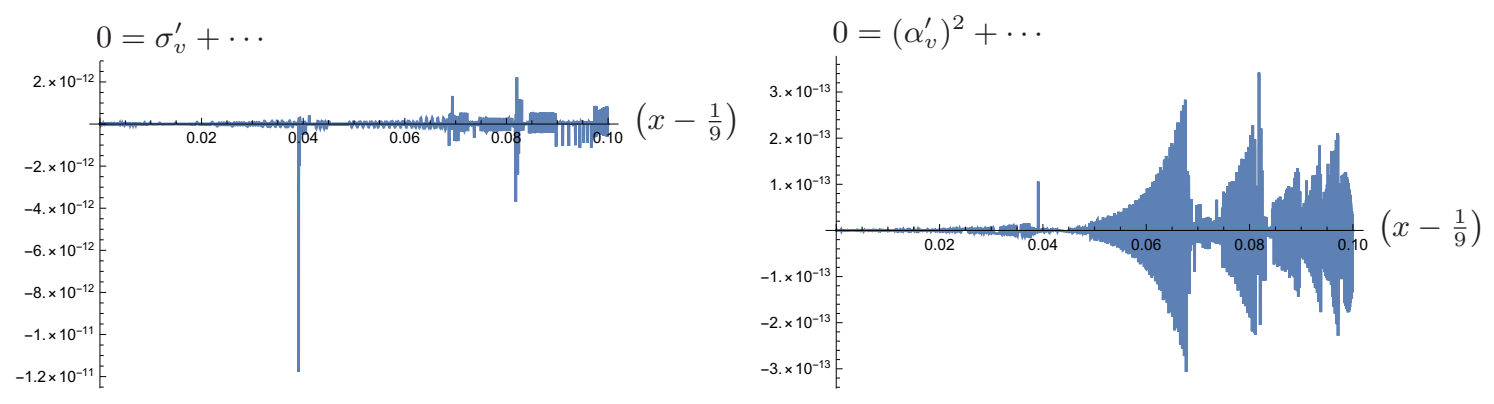

Figure 5. Same as in figure 4, but with $m_{b}=m_{f}=H$.
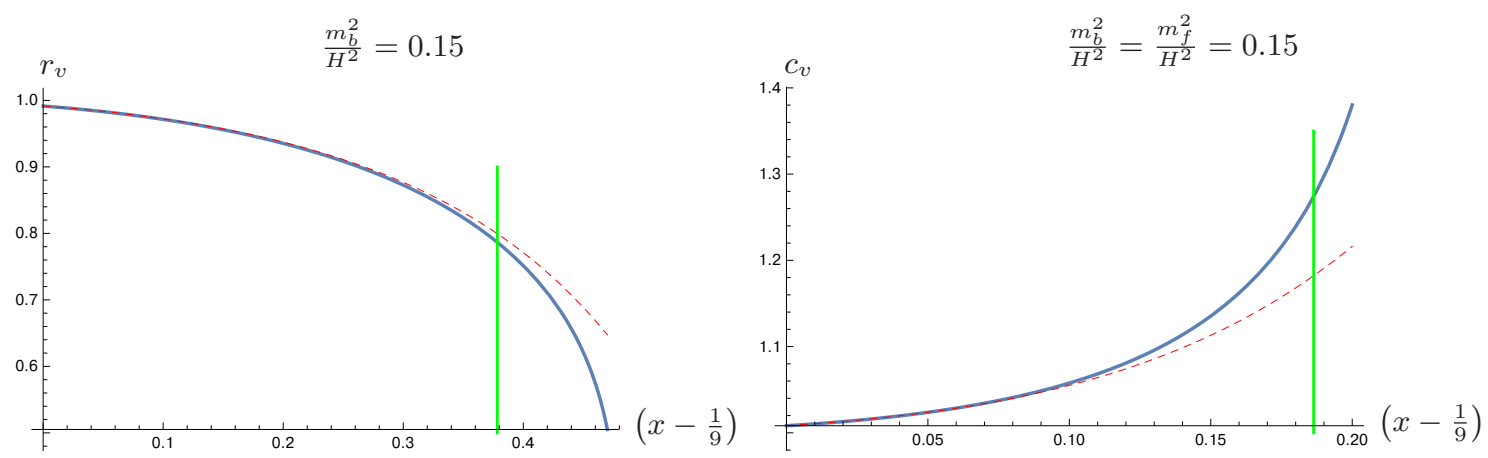

Figure 6. Profiles of the gravitational scalars $\left\{r_{0}, c_{0}\right\}$ (solid curves) describing the holographic dual to $\mathcal{N}=2^{*}$ gauge theory vacuum in $d S_{4}$ for select values of $\left\{m_{b}, m_{f}\right\}$ for radial coordinate $x>x_{s}=\frac{1}{9}$. The dashed red lines represent the analytic solutions valid for $\left\{m_{b}, m_{f}\right\} \ll H$, see (4.31) and (4.32). Vertical green lines indicate the location of the apparent horizon in the dual gravitational background.

$m_{b}=m_{f}=H$. The right panel of figure 7 presents the location of the apparent horizon $x_{\mathrm{AH}}$ in the gravitational dual to $\mathcal{N}=2^{*} d S_{4}$ vacuum at $m_{b}=m_{f}$.

Once we identify the location of the apparent horizon in the gravitational dual, we can compute the comoving entropy density production rate $\mathcal{R}$ of the theory in $d S_{4}$ vacuum, as defined in (4.10), using (3.17) as $t \rightarrow \infty$,

$$
3 H \times \mathcal{R}=\left.\frac{1}{H^{3}} \frac{2 N^{2}}{\pi}\left(\sigma_{v}^{3}\right)^{\prime} \frac{\left(A_{v} \chi_{v}^{\prime}\right)^{2}+\left(A_{v} \alpha_{v}^{\prime}\right)^{2}}{-4 \mathcal{P}}\right|_{r=r_{\mathrm{AH}}} .
$$




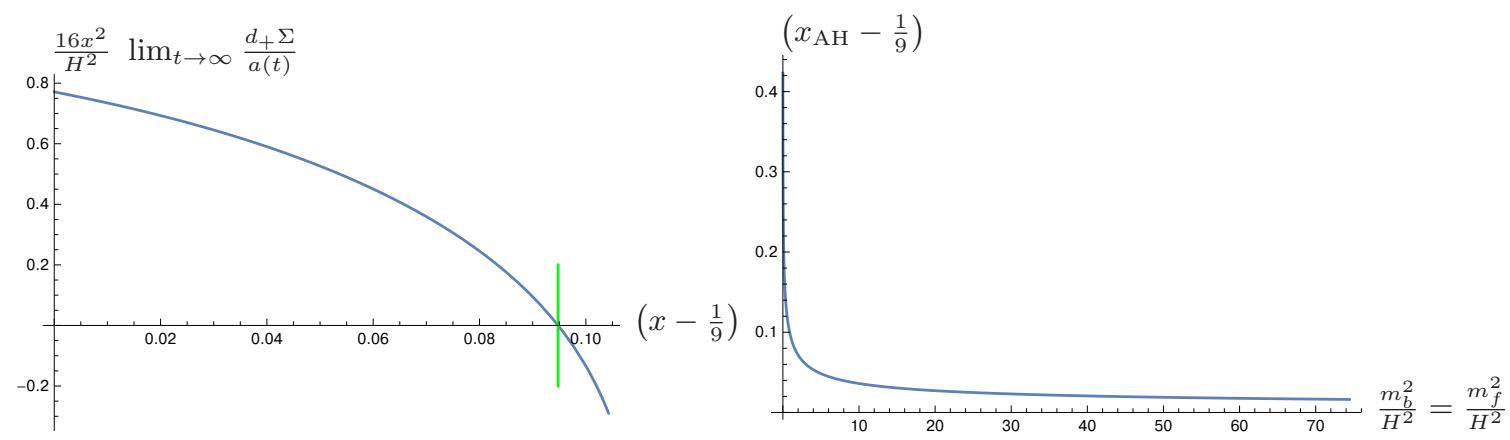

Figure 7. Left panel: apparent horizon of the $\mathcal{N}=2^{*}$ vacuum is determined from (4.9). The plot represents the $d_{+} \Sigma$ profile of the gravitational dual for $m_{b}=m_{f}=H$. Vertical green line indicates the location of the apparent horizon. Right panel: location of the apparent horizon $x_{\mathrm{AH}}$ as a function of $m_{b}=m_{f}$.
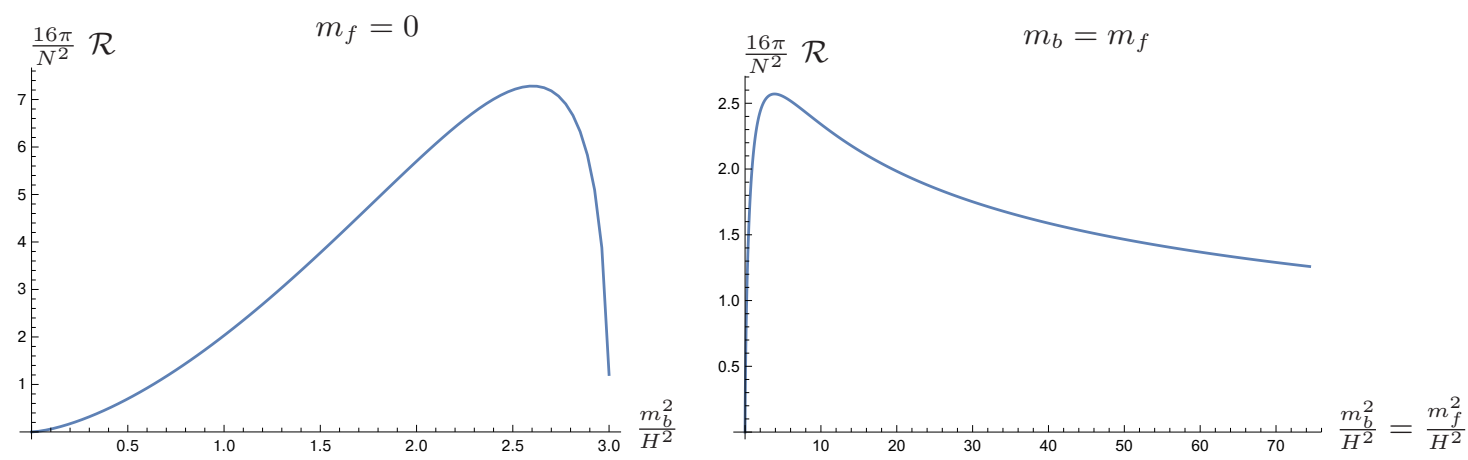

Figure 8. Comoving entropy production rate $\mathcal{R}(4.10)$ of $\mathcal{N}=2^{*}$ gauge theory in the $d S_{4}$ vacuum for select values of $\left\{m_{b}, m_{f}\right\}$.

Alternatively, we can use (3.16) to deduce

$$
\mathcal{R}=\left.\frac{1}{H^{3}} \frac{N^{2}}{16 \pi}\left(\sigma_{v}\right)^{3}\right|_{r=r_{\mathrm{AH}}} .
$$

Both formulas should agree - in fact, we find that the relative difference between the two results over the range of the mass parameters studied is $\lesssim 10^{-10}$. This excellent agreement is expected given the consistency checks on the numerics presented in figures $4-5$. Results for the comoving entropy production rate $\mathcal{R}$ are presented in figure 8 .

\section{Conclusion}

While a full understanding of quantum gravity is lacking, inflationary models can be improved with quantum treatment of the Standard Model gauge theories in classical gravitational backgrounds. This approximation is justified when there is a large hierarchy between the relevant QFT scales and the Planck scale. Thus, one needs to understand a QFT stress-energy tensor in curved space-time, see (1.1). This subject has a long history: although it is a textbook material [5], there are many unsolved problems, even in the case of weakly-coupled theories. 
In this paper we build up on the early observation [7] that when applicable, the holographic gauge/gravity correspondence $[3,4]$ can be used to achieve this goal. The Standard Model gauge theories do not have weakly coupled holographic gravitational dual; however, explicit holographic models can be studied to deduce the features of strongly coupled gauge theories in curved space-time. Here, we used the $\mathcal{N}=2^{*}$ holography to, hopefully, extract the general properties of quantum gauge theories in $d S_{4}$ space-time.

We summarize now our main observations.

- We argued that an initial state of the gauge theory in $d S_{4}$ would evolve to a universal static state which is a Bunch-Davies (Euclidean) vacuum. It does not appear possible to holographically evolve to a MA-vacuum $[16,17]$. This supports the claims in the literature [19-22] that interactions in QFTs invalidate the free-field construction of MA-vacua.

- We presented a holographic analog of the Weyl transformation between the Minkowski and the FLRW space-times. A conformal theory, up to computable anomalies, is invariant under this Weyl transformation; for a non-conformal theory one can map FLRW dynamics to Minkowski dynamics, provided the coupling constants of the relevant operators in the theory are appropriately "quenched" - prescribed a particular time-dependence.

- Holography provides a natural concept of entropy of a system far from equilibrium. Specifically, one identifies the entropy of the boundary gauge theory with the Bekenstein-Hawking entropy of the apparent horizon in the gravitational dual [39, 40]. Apparent horizon is observer dependent, however, in holographic setting describing dynamical evolution of spatially homogeneous and isotropy states of the boundary theory, there is a "preferred slicing" of the dual geometry. When the dynamical evolution of the gauge theory is hydrodynamic, the non-equilibrium entropy, defined in this manner, correctly reproduces Landau entropy [43]. We proved the theorem that even far from equilibrium, entropy associated with the apparent horizon of the gravitational holographic dual does not decrease with time. We also showed that the comoving entropy production rate computed with respect to a conformal time is invariant under Weyl transformations - there is no 'conformal anomaly' for the entropy transformations.

- Perhaps most surprisingly, we discovered that while the Bunch-Davies vacuum of a CFT is adiabatic - there is no comoving entropy production, a non-conformal gauge theory has a constant physical entropy density in its vacuum state; as a result the comoving entropy density grows with the background geometry scale factor as $a(t)^{3}$.

- One of the motivations of the project was an explicit computation of the gauge theory vacuum energy density $\mathcal{E}_{v}$, to address the feasibility of self-consistent and reliable accelerated expansion of the Universe, driven by the latter. Here, 'reliable' means that the solution of the Friedmann equation (1.6) produces the Hubble constant $H \ll M_{p l}$. Results for the vacuum energy density computation for $\mathcal{N}=2^{*}$ gauge 
theory at strong coupling are presented in figures 2-3. Much as it is in the case for the computation of the stress-energy tensor of weakly coupled QFTs in curved spacetime, $\mathcal{E}_{v}$ is prone to renormalization scheme ambiguities. The necessary condition for the accelerated expansion, i.e., $\mathcal{E}_{v}>0$ can be claimed in a scheme-independent way only for small values of the mass parameters $\left\{m_{b}, m_{f}\right\} \lesssim H$. However, in this case we are close to a conformal fixed point $(\mathcal{N}=4$ SYM in this case), and Friedmann equation produces $H \sim M_{p l}$. To conclude, $\mathcal{N}=2^{*}$ gauge theory at strong coupling can not produce accelerated expansion of the Universe with classical gravity.

Can the 'reliability' aspect of the accelerated expansion of the Universe from the $\mathcal{N}=$ $2^{*}$ vacuum energy discussed be fixed? Let's speculate that the vacuum energy of a QFT is (motivated by $(4.25)$ )

$\mathcal{E}_{v}(H)=Q_{1}^{2} H^{4}+\left(Q_{2}\left(m_{b}^{4}-m_{f}^{4}\right)-Q_{3}^{2} m_{f}^{2} H^{2}\right) \ln \frac{H}{\Lambda}+q_{1} m_{f}^{2} H^{2}+q_{2} m_{f}^{4}+q_{3} m_{b}^{2} H^{2}+q_{4} m_{b}^{4}$,

where $Q_{i}$ are fixed constants, and $\Lambda$ and $q_{i}$ encode scheme dependence. Basically, (5.1) maintains the rough features of the supersymmetry of the $\mathcal{N}=2^{*}$ model (whether $m_{b}=m_{f}$ or $m_{b} \neq m_{f}$ ), the ultraviolet conformal fixed point contribution $\left(Q_{1}^{2} H^{4}\right.$ term ), and the structure of the scheme dependence. What is different from the $\mathcal{N}=2^{*}$ model is the absence of contributions to the vacuum energy from the condensates of the relevant operators (the $r_{2,0}$ and $c_{4,0}$ terms in (4.25)). Let's analyze (5.1) in the context of the Friedmann equation (1.6). We are looking for a solution to (1.6) with $H \ll M_{p l}$. The renormalization scale $\Lambda$ is a QFT feature, so it is natural to have $\Lambda \ll M_{p l}$. There is no canonical way to define the energy density of a non-supersymmetric QFT - this ambiguity is reflected in $q_{2}$ and $q_{4}$ coefficients; however, if the theory is supersymmetric in Minkowski space-time, one expects that

$$
\left.\lim _{H \rightarrow 0} \mathcal{E}_{v}(H)\right|_{m_{b}=m_{f}=m}=0,
$$

provided the $H \rightarrow 0$ limit is smooth. Note that this limit is indeed smooth with $m_{b}=m_{f}$. Restricting to supersymmetric theories in Minkowski, we thus require $q_{2}+q_{4}=0$. Thus,

$$
\mathcal{E}_{v}^{\text {susy }}(H)=Q_{1}^{2} H^{4}+\left(-Q_{3}^{2} m^{2} H^{2}\right) \ln \frac{H}{\Lambda}+\left(q_{1}+q_{3}\right) m^{2} H^{2} .
$$

It is not possible to further fix the ambiguity associated with $\left(q_{1}+q_{3}\right)$ - even for a Euclidean supersymmetric formulation of gauge theories on $S^{4}$ such a term can not be fixed [34]. Interestingly, if $H \ll \Lambda$, the renormalization scheme sensitivity can be made arbitrarily weak. Moreover, if $H \ll m$, the conformal fixed point contribution is also irrelevant. The latter statement is precise if we choose the renormalization scale of the theory $\Lambda=m$. Thus,

$$
\mathcal{E}_{v}^{\text {susy }}(H) \approx-Q_{3}^{2} m^{2} H^{2} \ln \frac{H}{\Lambda}, \quad H \ll m \ll M_{p l} .
$$


Solving the Friedmann equation (1.6) with the vacuum energy density (5.3) we find

$$
H=m \exp \left[-\frac{3 M_{p l}^{2}}{8 \pi Q_{3}^{2} m^{2}}\right]
$$

naturally enforcing the hierarchy of scales in (5.3). As we emphasized, unfortunately, the $\mathcal{N}=2^{*}$ holographic model is not within the class of models with the energy density given by (5.1) - in this theory in the limit $m \gg H$ the contribution of the condensates of the relevant operators become important. In fact, precisely for $m_{b}=m_{f}$ case it can be shown that for $\frac{m}{H} \rightarrow \infty$ the effective coefficient $Q_{3}$ in $\mathcal{N}=2^{*}$ model vanishes.

It is interesting to further develop the dynamics of gauge theories in de Sitter. What replaces the 'hydrodynamics' in the approach of a QFT to its Bunch-Davies vacuum? What is the microscopic origin of the de Sitter entropy density of non-conformal theories? Do holographic models in the class (5.1) exist? We hope to address these questions in the future.

\section{Acknowledgments}

Research at Perimeter Institute is supported by the Government of Canada through Industry Canada and by the Province of Ontario through the Ministry of Research \& Innovation. This work was further supported by NSERC through the Discovery Grants program (AB).

\section{A Apparent horizon area growth theorem}

We prove here that the area density of the apparent horizon $\mathcal{A}_{\mathrm{AH}}$, see (3.16),

$$
\left.\mathcal{A}_{\mathrm{AH}}(t) \equiv \Sigma(t, r)^{3}\right|_{r=r_{\mathrm{AH}}},
$$

in a gravitational dual to a dynamical evolution of the boundary $\mathcal{N}=2^{*}$ gauge theory state does not decrease with the boundary time $t$,

$$
\frac{d \mathcal{A}_{\mathrm{AH}}}{d t} \geq 0
$$

This theorem implies that the comoving entropy density of the boundary gauge theory state, identified with the the Bekenstein-Hawking entropy density of the apparent horizon would satisfy the second law of thermodynamics.

Recall that (3.17)

$$
\frac{d \mathcal{A}_{\mathrm{AH}}}{d t}=\left.8\left(\Sigma^{3}\right)^{\prime} \frac{\left.\left(d_{+} \chi\right)^{2}+3\left(d_{+} \alpha\right)^{2}\right)}{-\mathcal{P}}\right|_{r=r_{\mathrm{AH}}} .
$$

Thus we need to establish two facts:

- $\Sigma^{\prime}\left(t, r_{A H}+0\right) \geq 0$

- $\left.\mathcal{P}\right|_{r=r_{\mathrm{AH}}+0} \leq 0$. 
Integrating the constraint equation (3.3) and using the boundary condition (3.5) we conclude

$$
\Sigma^{\prime}(t, r)=\frac{a}{2}+\int_{r}^{\infty} d \rho \frac{4}{3} \Sigma(t, \rho)\left(\left(\frac{\partial \chi(t, \rho)}{\partial \rho}\right)^{2}+3\left(\frac{\partial \alpha(t, \rho)}{\partial \rho}\right)^{2}\right) .
$$

Since $\Sigma(t, r)>0$ during the evolution, $\Sigma^{\prime}(t, r) \geq 0$.

Apparent horizon is defined as the innermost (with respect to the boundary) radial coordinate location $r=r_{\mathrm{AH}}$, where $d_{+} \Sigma(t, r)$ vanishes. Note from (3.5) that

$$
d_{+} \Sigma(t, r)=\frac{a r^{2}}{16}+\mathcal{O}\left(r^{0}\right)>0, \quad r \rightarrow \infty .
$$

Thus, assuming analyticity of the background geometry,

$$
d_{+} \Sigma(t, r)>0, \quad r>\left.r_{\mathrm{AH}} \quad \Longrightarrow \quad d_{+}^{\prime} \Sigma(t, r)\right|_{r=r_{\mathrm{AH}}} \geq 0
$$

The first evolution equation in (3.2) evaluated at the apparent horizon reads

$$
0=d_{+}^{\prime} \Sigma+\left.\frac{\Sigma}{6} \mathcal{P}\right|_{r=r_{\mathrm{AH}}+0} \quad \Longrightarrow \quad \mathcal{P}_{r=r_{\mathrm{AH}}+0} \leq 0
$$

\section{B Mapping QFT dynamics in Minkowski and FLRW space-times}

We argue that holographic QFT dynamics of homogeneous and isotropic states in Friedmann-Lemaitre-Robertson-Walker (FLRW) Universe

$$
d s^{2}=-d t^{2}+a(t)^{2} d \boldsymbol{x}^{2},
$$

is equivalent to a quenched dynamics [38] of the same theories in Minkowski space-time

$$
d \hat{s}^{2}=-d \tau^{2}+d \boldsymbol{x}^{2}, \quad \tau=\int_{0}^{t} \frac{d \nu}{a(\nu)},
$$

where any relevant ${ }^{8}$ coupling constant $\lambda_{\Delta}$ of dimension $\Delta<4$ operator $\mathcal{O}_{\Delta}$ being replaced with the corresponding time-dependent coupling $\hat{\lambda}_{\Delta}$ according to

$$
\lambda_{\Delta} \rightarrow \hat{\lambda}_{\Delta}(\tau)=a(t(\tau))^{4-\Delta} \lambda_{\Delta}
$$

The proof is elementary - while we implicitly focus on $\mathcal{N}=2^{*}$ model of holography, it is easily generalized to other holographic dualities, including those with gravitational bulk gauge fields (corresponding to charged states of the boundary gauge theory). The only requirement is that the QFT has an ultraviolet conformal fixed point. Indeed, the gravitational metric dual to QFT dynamics of homogeneous and isotropic states is given by (3.1), with the bulk scalars $\phi_{\Delta}=\phi_{\Delta}(t, r)$, dual to operators $\mathcal{O}_{\Delta}$. The asymptotic boundary

\footnotetext{
${ }^{8}$ It is possible to extend the result to marginal couplings as well.
} 
expansion encodes the background gauge theory metric and the coupling constants $\lambda_{\Delta}$ (compare with (3.5)):

$$
\Sigma=\frac{a(t) r}{2}+\mathcal{O}\left(r^{-1}\right), \quad A=\frac{r^{2}}{8}-\frac{\dot{a}(t) r}{a(t)}+\mathcal{O}\left(r^{0}\right), \quad \phi_{\Delta}=\frac{\lambda_{\Delta}}{r^{4-\Delta}}+\mathcal{O}\left(r^{5-\Delta}\right),
$$

where we used the fact that QFT is a four-dimensional CFT in the ultraviolet. Holographic equations of motion - the analog of (3.2)-(3.4) - come from the diffeomorphism invariant effective action in five dimensions. Thus, they are covariant under arbitrary change of bulk coordinates

$$
\{t, r\} \rightarrow\{\tau(t, r), \rho(t, r)\}
$$

Let's choose

$$
\tau \equiv \int_{0}^{t} \frac{d \nu}{a(\nu)}, \quad \rho \equiv r a(t)
$$

and introduce $\left\{\hat{\Sigma}, \hat{A}, \hat{\phi}_{\Delta}\right\}$ as

$$
\begin{aligned}
\hat{\Sigma}(\tau, \rho) & \equiv \Sigma(t(\tau), \rho / a(t(\tau))) \\
\hat{A}(\tau, \rho) & \equiv a(t(\tau))^{2} A(t(\tau), \rho / a(t(\tau)))+\frac{\rho}{a(t(\tau))} \frac{d}{d \tau} a(t(\tau)), \\
\hat{\phi}_{\Delta}(\tau, \rho) & \equiv \phi_{\Delta}(t(\tau), \rho / a(t(\tau))) .
\end{aligned}
$$

We emphasize that (B.6) and (B.7) is nothing but the change of coordinates, accompanied by field redefinitions. While mathematically trivial, it has profound implications physically: transformation (B.7) reparameterizes the background metric (3.1) $d s_{5}^{2}$ as

$$
d s_{5}^{2} \rightarrow d \hat{s}_{5}^{2} \equiv d s_{5}^{2}=2 d \tau(d \rho-\hat{A} d \tau)+\hat{\Sigma}^{2} d \boldsymbol{x}^{2},
$$

and produces a new set of the boundary conditions

$$
\hat{\Sigma}=\frac{\rho}{2}+\mathcal{O}\left(\rho^{-1}\right), \quad \hat{A}=\frac{\rho^{2}}{8}+\mathcal{O}\left(\rho^{0}\right), \quad \hat{\phi}_{\Delta}=\frac{\hat{\lambda}_{\Delta}(\tau)}{\rho^{4-\Delta}}+\mathcal{O}\left(\rho^{5-\Delta}\right),
$$

where $\hat{\lambda}_{\Delta}(\tau)$ is given by (B.3). (B.8) and (B.9) imply that the transformed holographic dynamics is that of the boundary gauge theory in Minkowski space-time (B.2) with quenched relevant couplings given by (B.3). Note that (B.7) applied at $\tau=t=0$ maps the initial states of the theories for equivalent dynamics in FLRW and $R^{3,1}$.

Interestingly, the map (B.7) leaves invariant the rate of the comoving entropy production computed with respect to the conformal time $d \tau=\frac{d t}{a(t)}$. Indeed, since

$$
\begin{aligned}
d_{+} & \equiv \partial_{t}+A(t, r) \partial_{r}=\frac{1}{a(t(\tau))}\left(\partial_{\tau}+\hat{A}(\tau, \rho) \partial_{\rho}\right)=\frac{1}{a(t(\tau))} \hat{d}_{+}, \\
\partial_{r} & =a(t(\tau)) \partial_{\rho},
\end{aligned}
$$

(3.18) implies that

$$
\frac{d\left(a^{3} s\right)}{d t}=\frac{1}{a(t(\tau))} \frac{d \hat{s}}{d \tau} .
$$

Notice that (B.11) establishes that equilibrium states of CFTs in Minkowski space-time are adiabatic in de Sitter. 


\section{C $\mathcal{N}=4 \mathrm{SYM}$ in FLRW}

We consider here a restrictive set of homogeneous and isotropic states of $\mathcal{N}=4 \mathrm{SYM}$ in FLRW space-time - the "thermal" states [29, 44]. These states are represented by solutions of holographic equations (3.2)-(3.4) with gravitational scalars $\alpha$ and $\chi$ identically set to zero.

The general solution of the holographic equations of motion within $\alpha=\chi \equiv 0$ ansatz takes form

$$
\begin{aligned}
& A=\frac{(r+\lambda(t))^{2}}{8}-(r+\lambda(t)) \frac{\dot{a}(t)}{a(t)}-\dot{\lambda}(t)-\frac{\mu^{4}}{8 a(t)^{4}(r+\lambda(t))^{2}}, \\
& \Sigma=\frac{(r+\lambda(t)) a(t)}{2},
\end{aligned}
$$

where $\lambda(t)$ is an arbitrary function related to the residual diffeomorphism invariance of the metric (3.1), and $\mu$ is a constant. Without loss of generality we assume that $\lambda<0$. From (3.15), the apparent horizon is then located at

$$
r_{\mathrm{AH}}=-\lambda(t)+\frac{\mu}{a(t)} .
$$

The comoving entropy density of the gauge theory is (see (3.16))

$$
a^{3} s=\frac{N^{2}}{16 \pi} \frac{\mu^{3}}{8} .
$$

Note that in agreement with (3.17), the comoving entropy density does not change with time; in particular, the entropy production rate $\mathcal{R}(4.10)$ in the vacuum state vanishes.

The adiabaticity of the evolution (C.1) can be understood from the equivalence theorem of appendix B. Choosing the gauge $\lambda(t)=0$ (as used in the derivation of the theorem) and applying the map (B.7), we find

$$
\hat{\Sigma}=\frac{\rho}{2}, \quad \hat{A}=\frac{\rho^{2}}{8}\left(1-\frac{\mu^{4}}{\rho^{4}}\right),
$$

which is the $A d S_{5}$ Schwarzschild black brane metric, dual to the equilibrium thermal state of the $\mathcal{N}=4 \mathrm{SYM}$. There is no entropy production in the thermal state; correspondingly, following the theorem of appendix B, there is no comoving entropy production in the state (C.1). It is for this reason we refer to the latter state as "thermal".

We would like to stress, as the $\mathcal{N}=4$ SYM example illustrates here, that even though the equivalence theorem of appendix B maps the dynamics of a QFT in FLRW to the one in Minkowski space-time, the energy density of the theory in Minkowski space-time is not the comoving energy density of the theory in FLRW:

$$
\hat{\mathcal{E}}=\frac{3}{8} \pi^{2} N^{2}\left(\frac{\mu}{4 \pi}\right)^{4}, \quad a(t)^{4} \mathcal{E}(t)=\hat{\mathcal{E}}+\frac{3 N^{2}(\dot{a}(t))^{4}}{32 \pi^{2}} .
$$

The difference between the two is due to the conformal anomaly in transformation from Minkowski to FLRW space-time [44]. 
Thermal states of $\mathcal{N}=4 \mathrm{SYM}$ in $d S_{4}$ at late times approach Bunch-Davies vacuum. Choosing the gauge $\lambda(t)=-H$ and redefining a radial coordinate as $r \equiv \frac{H}{x}, x \in\left(0, x_{\mathrm{AH}}\right]$ corresponding to $r \in\left[r_{\mathrm{AH}}, \infty\right)$, at late times $t \rightarrow \infty$, we find

$$
\begin{aligned}
x_{\mathrm{AH}} & \rightarrow 1, \\
\frac{\Sigma}{a} & \rightarrow \sigma_{v}=\frac{H}{2 x}(1-x), \\
A & \rightarrow A_{v}=\frac{H^{2}}{8 x^{2}}(1-x)(1-9 x) .
\end{aligned}
$$

Notice $^{9}$ that $A_{v}$ vanishes at

$$
x_{s}=\frac{1}{9} \in\left(0, x_{\mathrm{AH}}\right], \quad A_{v}\left(x_{s}\right)=0 .
$$

The $\mathcal{N}=4 \mathrm{SYM} d S_{4}$ vacuum energy is independent of the initial energy density, and is completely determined by the conformal anomaly:

$$
\frac{32 \pi^{2}}{N^{2}} \frac{\mathcal{E}_{v}}{H^{4}}=3 .
$$

Open Access. This article is distributed under the terms of the Creative Commons Attribution License (CC-BY 4.0), which permits any use, distribution and reproduction in any medium, provided the original author(s) and source are credited.

\section{References}

[1] Supernova Search Team collaboration, A.G. Riess et al., Observational evidence from supernovae for an accelerating universe and a cosmological constant, Astron. J. 116 (1998) 1009 [astro-ph/9805201] [INSPIRE].

[2] Supernova Cosmology Project collaboration, S. Perlmutter et al., Measurements of Omega and Lambda from 42 high redshift supernovae, Astrophys. J. 517 (1999) 565 [astro-ph/9812133] [INSPIRE].

[3] J.M. Maldacena, The large- $N$ limit of superconformal field theories and supergravity, Int. J. Theor. Phys. 38 (1999) 1113 [hep-th/9711200] [INSPIRE].

[4] O. Aharony, S.S. Gubser, J.M. Maldacena, H. Ooguri and Y. Oz, Large-N field theories, string theory and gravity, Phys. Rept. 323 (2000) 183 [hep-th/9905111] [INSPIRE].

[5] N.D. Birrell and P.C.W. Davies, Quantum Fields in Curved Space, Cambridge University Press (1982), doi:10.1017/CBO9780511622632.

[6] S.W. Hawking, T. Hertog and H.S. Reall, Trace anomaly driven inflation, Phys. Rev. D 63 (2001) 083504 [hep-th/0010232] [InSPIRE].

[7] A. Buchel, Gauge/gravity correspondence in accelerating universe, Phys. Rev. D 65 (2002) 125015 [hep-th/0203041] [INSPIRE].

[8] A. Buchel, P. Langfelder and J. Walcher, On time dependent backgrounds in supergravity and string theory, Phys. Rev. D 67 (2003) 024011 [hep-th/0207214] [INSPIRE].

\footnotetext{
${ }^{9}$ See discussion following (4.9).
} 
[9] A. Buchel, Compactifications of the $N=2^{*}$ flow, Phys. Lett. B 570 (2003) 89 [hep-th/0302107] [INSPIRE].

[10] A. Buchel and A. Ghodsi, Braneworld inflation, Phys. Rev. D 70 (2004) 126008 [hep-th/0404151] [INSPIRE].

[11] A. Buchel, Inflation on the resolved warped deformed conifold, Phys. Rev. D 74 (2006) 046009 [hep-th/0601013] [INSPIRE].

[12] A. Buchel and D.A. Galante, Cascading gauge theory on $d S_{4}$ and String Theory landscape, Nucl. Phys. B 883 (2014) 107 [arXiv:1310.1372] [INSPIRE].

[13] L. Anguelova, P. Suranyi and L.C.R. Wijewardhana, De Sitter Space in Gauge/Gravity Duality, Nucl. Phys. B 899 (2015) 651 [arXiv: 1412.8422] [INSPIRE].

[14] L. Anguelova, A Gravity Dual of Ultra-slow Roll Inflation, Nucl. Phys. B 911 (2016) 480 [arXiv: 1512.08556] [INSPIRE].

[15] T.S. Bunch and P.C.W. Davies, Quantum Field Theory in de Sitter Space: Renormalization by Point Splitting, Proc. Roy. Soc. Lond. A 360 (1978) 117.

[16] E. Mottola, Particle Creation in de Sitter Space, Phys. Rev. D 31 (1985) 754 [INSPIRE].

[17] B. Allen, Vacuum States in de Sitter Space, Phys. Rev. D 32 (1985) 3136 [InSPIRE].

[18] R. Easther, B.R. Greene, W.H. Kinney and G. Shiu, Inflation as a probe of short distance physics, Phys. Rev. D 64 (2001) 103502 [hep-th/0104102] [inSPIRE].

[19] T. Banks and L. Mannelli, De Sitter vacua, renormalization and locality, Phys. Rev. D 67 (2003) 065009 [hep-th/0209113] [INSPIRE].

[20] M.B. Einhorn and F. Larsen, Interacting quantum field theory in de Sitter vacua, Phys. Rev. D 67 (2003) 024001 [hep-th/0209159] [INSPIRE].

[21] M.B. Einhorn and F. Larsen, Squeezed states in the de Sitter vacuum, Phys. Rev. D 68 (2003) 064002 [hep-th/0305056] [INSPIRE].

[22] H. Collins, R. Holman and M.R. Martin, The fate of the $\alpha$-vacuum, Phys. Rev. D 68 (2003) 124012 [hep-th/0306028] [INSPIRE].

[23] K. Pilch and N.P. Warner, $N=2$ supersymmetric $R G$ flows and the IIB dilaton, Nucl. Phys. B 594 (2001) 209 [hep-th/0004063] [INSPIRE].

[24] A. Buchel, A.W. Peet and J. Polchinski, Gauge dual and noncommutative extension of an $N=2$ supergravity solution, Phys. Rev. D 63 (2001) 044009 [hep-th/0008076] [INSPIRE].

[25] N.J. Evans, C.V. Johnson and M. Petrini, The enhancon and $N=2$ gauge theory: Gravity $R G$ flows, JHEP 10 (2000) 022 [hep-th/0008081] [INSPIRE].

[26] I.R. Klebanov and M.J. Strassler, Supergravity and a confining gauge theory: Duality cascades and chi SB resolution of naked singularities, JHEP 08 (2000) 052 [hep-th/0007191] [INSPIRE].

[27] E. Witten, Anti-de Sitter space, thermal phase transition and confinement in gauge theories, Adv. Theor. Math. Phys. 2 (1998) 505 [hep-th/9803131] [InSPIRE].

[28] O. Aharony, A. Buchel and A. Yarom, Holographic renormalization of cascading gauge theories, Phys. Rev. D 72 (2005) 066003 [hep-th/0506002] [INSPIRE]. 
[29] A. Buchel, M.P. Heller and J. Noronha, Entropy production, hydrodynamics and resurgence in the primordial quark-gluon plasma from holography, Phys. Rev. D 94 (2016) 106011 [arXiv: 1603.05344] [INSPIRE].

[30] R. Donagi and E. Witten, Supersymmetric Yang-Mills theory and integrable systems, Nucl. Phys. B 460 (1996) 299 [hep-th/9510101] [INSPIRE].

[31] A. Buchel, J.G. Russo and K. Zarembo, Rigorous Test of Non-conformal Holography: Wilson Loops in $N=2^{*}$ Theory, JHEP 03 (2013) 062 [arXiv:1301.1597] [INSPIRE].

[32] A. Buchel, Localization and holography in $N=2$ gauge theories, JHEP 08 (2013) 004 [arXiv: 1304.5652] [INSPIRE].

[33] V. Pestun, Localization of gauge theory on a four-sphere and supersymmetric Wilson loops, Commun. Math. Phys. 313 (2012) 71 [arXiv:0712.2824] [InSPIRE].

[34] N. Bobev, H. Elvang, D.Z. Freedman and S.S. Pufu, Holography for $N=2^{*}$ on $S^{4}$, JHEP 07 (2014) 001 [arXiv: 1311.1508] [INSPIRE].

[35] V. Balasubramanian and A. Buchel, On consistent truncations in $N=2^{*}$ holography, JHEP 02 (2014) 030 [arXiv: 1311.5044] [INSPIRE].

[36] P.M. Chesler and L.G. Yaffe, Numerical solution of gravitational dynamics in asymptotically anti-de Sitter spacetimes, JHEP 07 (2014) 086 [arXiv:1309.1439] [INSPIRE].

[37] A. Buchel, S. Deakin, P. Kerner and J.T. Liu, Thermodynamics of the $N=2^{*}$ strongly coupled plasma, Nucl. Phys. B 784 (2007) 72 [hep-th/0701142] [INSPIRE].

[38] A. Buchel, L. Lehner and R.C. Myers, Thermal quenches in $N=2^{*}$ plasmas, JHEP 08 (2012) 049 [arXiv:1206.6785] [INSPIRE].

[39] I. Booth, Black hole boundaries, Can. J. Phys. 83 (2005) 1073 [gr-qc/0508107] [InSPIRE].

[40] P. Figueras, V.E. Hubeny, M. Rangamani and S.F. Ross, Dynamical black holes and expanding plasmas, JHEP 04 (2009) 137 [arXiv: 0902.4696] [INSPIRE].

[41] A. Buchel, R.C. Myers and A. van Niekerk, Nonlocal probes of thermalization in holographic quenches with spectral methods, JHEP 02 (2015) 017 [Erratum ibid. 1507 (2015) 137] [arXiv: 1410.6201] [INSPIRE].

[42] O. Aharony, A. Buchel and P. Kerner, The black hole in the throat: Thermodynamics of strongly coupled cascading gauge theories, Phys. Rev. D 76 (2007) 086005 [arXiv:0706.1768] [INSPIRE].

[43] L.D. Landau and E.M. Lifshitz, Course of Theoretical Physics, Volume 6: Fluid Mechanics, Pergamon Press (1959).

[44] P.S. Apostolopoulos, G. Siopsis and N. Tetradis, Cosmology from an AdS Schwarzschild black hole via holography, Phys. Rev. Lett. 102 (2009) 151301 [arXiv:0809.3505] [INSPIRE]. 